\title{
THE BLANCHET-KHOVANOV ALGEBRAS
}

\author{
MICHAEL EHRIG, CATHARINA STROPPEL, AND DANIEL TUBBENHAUER
}

Dedicated to Christian Blanchet's sixtieth birthday

\begin{abstract}
Blanchet introduced certain singular cobordisms to fix the functoriality of Khovanov homology. In this paper we introduce graded algebras consisting of such singular cobordisms à la Blanchet. As the main result we explicitly describe these algebras in algebraic terms using the combinatorics of arc diagrams.
\end{abstract}

\section{Contents}

1. Introduction

2. $\mathfrak{g l}_{2}$-foams and $\mathfrak{g l}_{2}$-web algebras

2.1. Webs, foams and TQFTs

2.2. Blanchet's singular TQFT construction

2.3. An action of the quantum group $\dot{\mathrm{U}}_{q}\left(\mathfrak{g l}_{\infty}\right)$

2.4. $\mathfrak{g l}_{2}$-web algebras

2.5. Web bimodules

3. Blanchet-Khovanov algebras

3.1. Combinatorics of arc diagrams

3.2. The Blanchet-Khovanov algebras as graded $\mathbb{K}$-vector spaces

3.3. Multiplication of the Blanchet-Khovanov algebra

3.4. Bimodules for Blanchet-Khovanov algebras

4. Equivalences

4.1. Some useful lemmas

4.2. An action of the quantum group $\dot{\mathrm{U}}_{q}\left(\mathfrak{g l}_{\infty}\right)$ and arc diagrams

4.3. The cup basis

4.4. Proof of the main result

4.5. The proof of the graded isomorphism

References

\section{InTRODUCTION}

For an arbitrary field $\mathbb{K}$ we consider the $\mathfrak{g l}_{2}$-web algebra $\mathfrak{W}$, which we call web algebra for short. (For the reason why we like to call it $\mathfrak{g l}_{2}$-web algebra instead of $\mathfrak{s l}_{2}$-web algebra see in the introduction of [14].) This is a graded $\mathbb{K}$-algebra which naturally appears as an algebra of singular cobordisms. In particular, it is of topological origin. The underlying category of singular cobordisms was used in [2] by Blanchet to fix the functoriality of Khovanov homology. Its objects are certain trivalent graphs and its morphisms are singular cobordisms whose boundary are such trivalent graphs. We call these singular cobordisms $\mathfrak{g l}_{2}$-foams (or foams for short). Note that Blanchet's category is a sign modified version of the original cobordism category which describes Khovanov homology and which was for instance used by Bar-Natan in his formulation of Khovanov homology, see [1. The fact that such a twist in the definition of Khovanov homology solves the functoriality leaves the question whether this could also be fixed algebraically using the original construction of Khovanov involving his arc algebra, see [18.

We therefore suggest here to study a certain signed (with highly non-trivial sign modifications) version $\mathfrak{A}^{\mathfrak{F}}$ of Khovanov's original algebra, which we call the 
Blanchet-Khovanov algebra. This is a graded $\mathbb{K}$-algebra defined diagrammatically via explicit multiplication rules on a distinguished set of basis vectors similar to the family of algebras from [4] or [12.

The main result of the paper is then that $\mathfrak{A}^{\mathfrak{F}}$ is an algebraic counterpart of $\mathfrak{W}$ :

Theorem. There is an equivalence of graded, $\mathbb{K}$-linear 2-categories

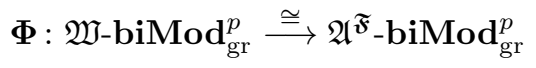

induced by an isomorphism of graded algebras

$$
\Phi: \mathfrak{W}^{\circ} \rightarrow \mathfrak{A}^{\mathfrak{F}} .
$$

(Where $\mathfrak{W}^{\circ}$ is a certain subalgebra of $\mathfrak{W}$.)

This provides a direct link between the topological and the algebraic point of view. As a consequence, computations (which are hard to do in practice on the topological side) can be done on the algebraic side, whereas the associativity (a non-trivial fact on the algebraic side) is clear from the topological point of view.

The set-up in more details. In his pioneering work [18, Khovanov introduced the so-called arc algebra $H_{m}$. One of his main purposes was to extend his celebrated categorification of the Jones polynomial [17] to tangles. To a given tangle with $2 m$ bottom boundary points and $2 m^{\prime}$ top boundary points one associates a certain complex of graded $H_{m}-H_{m^{\prime}}$-bimodules. He showed that the chain homotopy equivalence class of this complex is an invariant of the tangle. Moreover, taking the tensor product with a certain $H_{m}$-module from the left and a certain $H_{m^{\prime}}$-module from the right produces a complex which is still an invariant. On the level of Grothendieck groups this invariant descends to the Kauffman bracket of the tangle.

In this set-up it makes sense to ask if cobordisms between tangles correspond to natural transformations between bimodules. Or said in other words, whether there is a 2-functor from the 2-category of tangles to a certain 2-category of $H=\bigoplus_{m \in \mathbb{Z}_{\geq 0}} H_{m}$-bimodules. This is often called functoriality.

In a series of papers [4, [5], 6], 7] and [8] a generalization $\mathfrak{A}_{\Lambda}$ of the arc algebra was studied revealing that Khovanov's arc algebra has, left aside its knot theoretical origin, interesting representation theoretical, algebraic geometrical and combinatorial properties. These algebras $\mathfrak{A}_{\Lambda}$ were defined using an algebraic approach via the combinatorics of arc diagrams, i.e. certain diagrams consisting of embedded lines in $\mathbb{R}^{2}$ inspired by the diagrams for Temperley-Lieb algebras.

This series of results has led to several variations and generalizations of Khovanov's original formulation, utilized in a large body of work by several researchers (including the authors of this paper), e.g. an $\mathfrak{s l}_{3}$-variation considered in [27, [32, [31] and [36], and an $\mathfrak{s l}_{n}$-variation studied in [26] and [37], all of them having relations to (cyclotomic) KL-R algebras as in [20] or [33, and link homologies in the sense of Khovanov and Rozansky [21]. There is also the $\mathfrak{g l}_{1 \mid 1}$-variation developed in [34] with relations to the Alexander polynomial as well as a type $\mathbf{D}$-version introduced in [11] and 12] with connections to the representation theory of Brauer's centralizer algebras and orthosymplectic Lie superalgebras, see e.g. [13.

A fact we like to stress about the $\mathfrak{s l}_{3} / \mathfrak{s l}_{n}$-variations is that their graded 2 -categories of biprojective, finite-dimensional modules are equivalent to certain graded 2 -categories of $\mathfrak{s l}_{3} / \mathfrak{s l}_{n}$-foams, the analogues of Bar-Natan's cobordism category [1] studied e.g. in [19], 24], 28] and [29] from the viewpoint of link homologies. (We note hereby that such a topological description for the type $\mathbf{D}$-version was found in [15], providing, in some sense, the first "foamy description" outside of type A.)

We like to stress that Khovanov's original construction as well as Bar-Natan's reformulation from [1] are not functorial, but are functorial up to signs, see [1], [16], [30] 
or 35. It became clear that Bar-Natan cobordisms miss some subtle extra signs (see for example [10] for the first fix of functoriality using "disoriented" cobordisms).

A solution to this problem, that is of key interest for us, was provided by Blanchet in [2]. He formulated Khovanov's link homology using certain singular cobordisms, that we call $\left(\mathfrak{g l}_{2^{-}}\right)$foams, which, by construction, include highly non-trivial signs fixing the functoriality of Khovanov's link homology. Moreover, Blanchet's formulation fits neatly into the framework of graded 2-representations of the categorified quantum group in the sense of [20], as it was shown in [24].

Using Blanchet's construction it makes sense to define "foamy" versions $\mathfrak{W}_{\vec{k}}$ of Khovanov's arc algebra, which we call $\left(\mathfrak{g l}_{2^{-}}\right)$web algebras. We set $\mathfrak{W}=\bigoplus_{\vec{k} \in \mathfrak{b l}_{\mathbb{1}} \diamond} \mathfrak{W}_{\vec{k}}$. The web algebras $\mathfrak{W}_{\vec{k}}$ and $\mathfrak{W}$ are graded $\mathbb{K}$-algebras defined using Blanchet's singular cobordisms and the multiplication is given by composition of singular cobordisms (for our conventions see Section 2). The signs within this multiplication are quite sophisticated, e.g. even merges (which are quite easy in the formulations of [4] and [12]) can come with a sign.

Unfortunately calculating in $\mathfrak{W}_{\vec{k}}$ and $\mathfrak{W}$ is very hard. Indeed, it is not even clear what a basis of $\mathfrak{W}_{\vec{k}}$ or $\mathfrak{W}$ is - left aside the question how to rewrite an arbitrary foam in terms of some basis. Thus, the main purpose of this paper is to give algebraic counterparts of $\mathfrak{W}_{\vec{k}}$ and $\mathfrak{W}$, denoted by $\mathfrak{A}_{\Lambda}^{\mathfrak{F}}$ and $\mathfrak{A}^{\mathfrak{F}}=\bigoplus_{\Lambda \in \mathrm{bl}} \mathfrak{A}_{\Lambda}^{\mathfrak{F}}$ where these questions about bases are easy. We call the algebraic counterparts, which are built up using certain combinatorics of arc diagrams, Blanchet-Khovanov algebras.

The proof of our main theorem relies on the rather subtle Theorem 4.18 which needs careful treatment of all involved signs. The whole Subsection 4.5 is devoted to its proof. Our main theorem clarifies algebraically the deficiency in the original theory. For brevity, we stop our investigation here although several natural questions remain open, e.g. a direct representation theoretic construction of Blanchet-Khovanov algebras, see Remark 4.20 .

To keep the paper self-contained we start by a rather detailed exposition of the main ingredients and players adapted to the main purpose of the paper.

Conventions used throughout.

Convention 1.1. Let $\mathbb{K}$ denote a field of arbitrary characteristic. (We sometimes need to work with $\mathbb{K}(q)$ for a formal parameter $q$. All notions below are similarly defined in this case.) An algebra always means a non-necessarily finite-dimensional, non-necessarily unital $\mathbb{K}$-algebra $A$. We do not assume that such $A$ 's are associative and it will be a non-trivial fact that all $A$ 's which we consider are actually associative. Given two algebras $A$ and $B$, then an $A$-B-bimodule is a $\mathbb{K}$-vector space $M$ with a left action of $A$ and a right action of $B$ in the usual sense. If $A=B$, then we also write $A$-bimodule for short. We call an $A$-B-bimodule $M$ biprojective, if it is projective as a left $A$-module and right $B$-module (such finitely generated bimodules are called sweet in [18, Subsection 2.6]). We denote the category of locally finite-dimensional $A$-bimodules by $A$-biMod, i.e. the category of $A$-bimodules $M$ such that $e M e^{\prime}$ is finite-dimensional for any two primitive idempotents $e, e^{\prime} \in A$. Diagrammatic left (or right) actions will be given by acting on the bottom (or top).

Convention 1.2. By a graded algebra we mean an algebra $A$ which decomposes into graded pieces $A=\bigoplus_{i \in \mathbb{Z}} A_{i}$ such that $A_{i} A_{j} \subset A_{i+j}$ for all $i, j \in \mathbb{Z}$. Given two graded algebras $A$ and $B$, we study (and only consider) graded $A$-B-bimodules, i.e. $A$-B-bimodules $M=\bigoplus_{i \in \mathbb{Z}} M_{i}$ such that $A_{i} M_{j} B_{k} \subset M_{i+j+k}$ for all $i, j, k \in \mathbb{Z}$. We also set $M_{i}\{s\}=M_{i-s}$ for $s \in \mathbb{Z}$ (thus, positive integers shift up).

If $A$ is a graded algebra and $M$ is a graded $A$-bimodule, then $\bar{M}$ obtained from $M$ by forgetting the grading is in $A$-biMod. 
Given such $A$-bimodules $\bar{M}, \bar{N}$, then

$$
\operatorname{Hom}_{A \text {-biMod }}(\bar{M}, \bar{N})=\bigoplus_{s \in \mathbb{Z}} \operatorname{Hom}_{0}(M, N\{s\}) .
$$

Here $\mathrm{Hom}_{0}$ means all degree-preserving $A$-homomorphisms, i.e. $f\left(M_{i}\right) \subset N_{i}$.

Convention 1.3. We consider three diagrammatic calculi in this paper: $\mathfrak{g l}_{2}$-webs (webs for short) in the sense of [9] and [23, foams whose definition is motivated from [2] and [19], and arc diagrams in the sense of [4] and [6]. Our reading convention for all of these is from bottom to top and from left to right. We often illustrate local pieces only; the diagram then is meant to be the identity or arbitrary outside of the displayed part (which one will be clear from the context).

Remark 1.4. We use colors in this paper. It is only necessary to distinguish colors for webs and foams. For the readers with a black-and-white version: we illustrate colored web edges using dashed lines, while colored foam facets appear shaded.

Acknowledgements: We like to thank David Rose and Nathalie Wahl for helpful conversations, and Paul Wedrich and the referee for helpful comments. M.E. and D.T. thank the whiteboard in their office for many helpful illustrations.

\section{2. $\mathfrak{g l}_{2}$-FOAMS AND $\mathfrak{g l}_{2}$-WEB ALGEBRAS}

In this section we introduce the foam 2-category $\mathfrak{F}$ and the web algebra $\mathfrak{W}$ in the spirit of Khovanov [18, but using foams à la Blanchet [2].

2.1. Webs, foams and TQFTs. We start by recalling the definition of a web. For this purpose, we denote by $\overline{\mathrm{b} \mathbb{1}}$ the set of all vectors $\vec{k}=\left(k_{i}\right)_{i \in \mathbb{Z}} \in\{0,1,-1,2,-2\}^{\mathbb{Z}}$ with $k_{i}=0$ for $|i| \gg 0$. Abusing notation, we also sometimes write $\vec{k}=\left(k_{a}, \ldots, k_{b}\right)$ for some fixed part of $\vec{k}$ (with $a<b \in \mathbb{Z}$ ) where it is to be understood that all non-displayed entries are zero. By convention, the empty vector is the unique vector containing only zeros. We consider $\vec{k} \in \overline{\mathrm{b} \mathbb{1}}$ as a set of discrete labeled points in $\mathbb{R} \times\{ \pm 1\}$ (or in $\mathbb{R} \times\{0\}$ ) by putting the symbols $k_{i}$ at position $(i, \pm 1)($ or $(i, 0)$ ). We denote by $\mathfrak{b l} \subset \overline{\mathrm{b} \mathbb{1}}$ the subset of all vectors with entries from $\{0,1,2\}$ only.

Definition 2.1. A web is an embedded labeled, oriented, trivalent graph which can be obtained by gluing (whenever this makes sense and the labels fit) or juxtaposition of finitely many (possibly zero) of the following pieces:

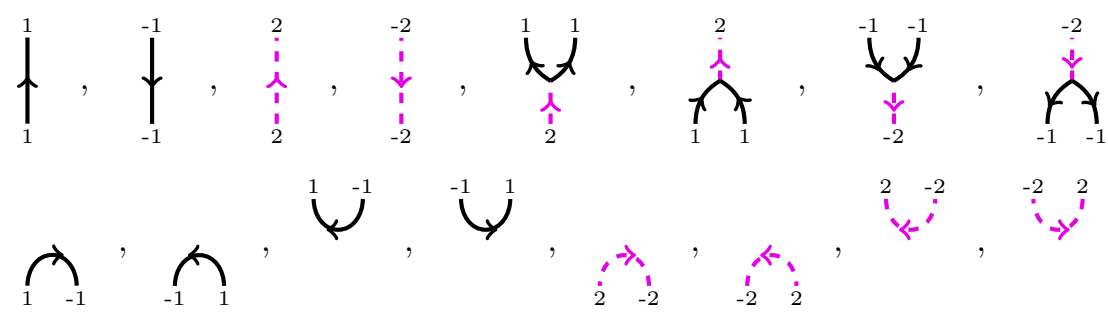

(Hence, this includes the empty web.) We assume that webs are embedded in $\mathbb{R} \times[-1,1]$ such that each edge starts/ends either in a trivalent vertex or at the boundary of the strip at the points $(i, \pm 1)$. We assume that the points at $(i, \pm 1)$ are labeled $1,-1,2$ or -2 . In particular, these webs have distinguished bottom $\vec{k}$ and top $\vec{l}$ boundary which we will throughout denote from left to right by $\vec{k}=\left(k_{a}, \ldots, k_{b}\right)$ and $\vec{l}=\left(l_{a^{\prime}}, \ldots, l_{b^{\prime}}\right)$ where $k_{i}$ is the label at $(i,-1)$ and $l_{i}$ is the label at $(i, 1)$.

Edges come in two different version, i.e. ordinary edges which are only allowed to have boundary points labeled 1 or -1 , and phantom edges which are only allowed to have boundary points labeled 2 or -2 . As in (2), we draw phantom edges dashed (and colored); one should think of them as "non-existing". 
We denote the set consisting of all webs with bottom boundary $\vec{k}$ and top boundary $\vec{l}$ by $\operatorname{Hom}_{\overline{\mathfrak{F}}}(\vec{k}, \vec{l})$ (for a reason that will become clear later). Given $\vec{k} \in \overline{\mathrm{b}} \mathbb{1}$, we denote by $\mathbf{1}_{\vec{k}} \in \operatorname{Hom}_{\overline{\mathfrak{F}}}(\vec{k}, \vec{k})$ the identity web on $\vec{k}$.

Remark 2.2. For our purposes it will be mostly sufficient to consider webs in a "highest weight setup", i.e. only upwards pointing webs (see also Subsection 2.3). In particular, we do not need the labels -1 and -2 much in this paper. Still, all construction from this and the next subsection can be done in a more flexible setup using topological webs, which are however not necessary for our purposes.

By a surface we mean a marked, orientable, compact surface with possible finitely many boundary components and with finitely many connected components. Additionally, by a trivalent surface we understand the same as in [19, Subsection 3.1], i.e. certain embedded, marked, singular cobordisms whose boundaries are webs.

Precisely, fix the following data denoted by $\boldsymbol{S}$ :

(I) A surface $S$ with connected components divided into two sets $\left\{S_{1}^{\mathrm{o}}, \ldots, S_{r}^{\mathrm{o}}\right\}$ and $\left\{S_{1}^{\mathrm{p}}, \ldots, S_{r^{\prime}}^{\mathrm{p}}\right\}$. (The former are called ordinary surfaces and the latter are called phantom surfaces.)

(II) The boundary components of $S$ are partitioned into triples $\left(C_{i}^{\mathrm{o}}, C_{j}^{\mathrm{o}}, C_{k}^{\mathrm{p}}\right)$ such that each triple contains precisely one boundary component $C_{k}^{\mathrm{p}}$ of a phantom surface.

(III) The three circles $C_{i}^{\mathrm{o}}, C_{j}^{\mathrm{o}}$ and $C_{k}^{\mathrm{p}}$ in each triple are identified via diffeomorphisms $\varphi_{i j}: C_{i}^{\mathrm{o}} \rightarrow C_{j}^{\mathrm{o}}$ and $\varphi_{j k}: C_{j}^{\mathrm{o}} \rightarrow C_{k}^{\mathrm{p}}$.

(IV) A finite (possible empty) set of markers per connected components.

Definition 2.3. Let $\boldsymbol{S}$ be as above. The closed, singular trivalent surface $f_{c}=f_{c}^{\boldsymbol{S}}$ attached to $\boldsymbol{S}$ is the $\mathrm{CW}$-complex obtained as the quotient of $S$ by the identifications $\varphi_{i j}$ and $\varphi_{j k}$. We call all such $f_{c}$ 's closed pre-foams (following [19]) and their markers dots. A triple $\left(C_{i}^{\mathrm{o}}, C_{j}^{\mathrm{o}}, C_{k}^{\mathrm{p}}\right)$ becomes one circle in $f_{c}$ which we call a singular seam, while the interior of the connected components $S_{1}^{\mathrm{o}}, \ldots, S_{r}^{\mathrm{o}}$ and $S_{1}^{\mathrm{p}}, \ldots, S_{r^{\prime}}^{\mathrm{p}}$ are facets of $f_{c}$, called ordinary facets respectively phantom facets. We embed these pre-foams into $\mathbb{R}^{3}$ in such a way that the three annuli glued to a singular seam can be oriented. We additionally choose orientations on the singular seams, compare to (3).

Example 2.4. Consider two spheres with two punctures respectively one puncture. The sphere with one puncture is assumed to be a phantom sphere (we color phantom facets in what follows).
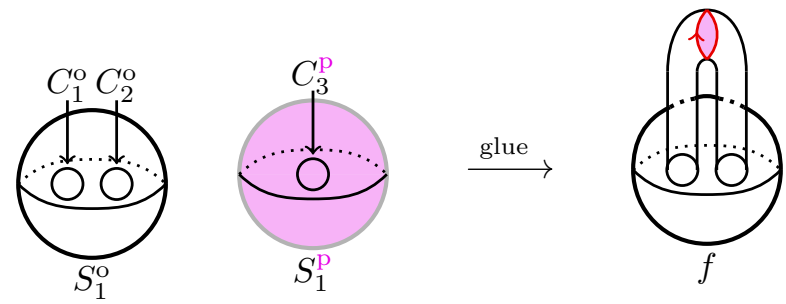

Then the pre-foam on the right is obtained by identifying the three boundary circles. (We have also chosen an orientation of the singular seam.) Another example of a closed pre-foam is given below in the proof of Lemma 2.13 (where we leave it to the reader to identify the precise labels).

The pre-foams we have constructed so far are all closed. We will need non-closed pre-foams as well. To this end, we follow [19, Subsection 3.3] and consider a plane $P \cong \mathbb{R}^{2} \subset \mathbb{R}^{3}$. We say that $P$ intersects a closed pre-foam $f_{c}$ generically, if $P \cap f_{c}$ is a non-oriented web (seen as a topological space). 
Definition 2.5. Let $P_{x y}^{ \pm 1}$ be the $x y$-plane in $\mathbb{R}^{3}$ (embedded such that the third coordinate is \pm 1 ). A (non-closed) pre-foam $f$ is the intersection of $\mathbb{R}^{2} \times[-1,1]$ with some closed pre-foam $f_{c}$ such that $P_{x y}^{ \pm 1}$ intersects $f_{c}$ generically, and the intersection is a web as in Definition 2.1. We see such pre-foam $f$ as a singular cobordism between $P_{x y}^{-1} \cap f_{c}$ (bottom, source) and $P_{x y}^{+1} \cap f_{c}$ (top, target) embedded in $\mathbb{R}^{2} \times[-1,1]$. Moreover, there is an evident composition $g \circ f$ via gluing and rescaling. Similarly, we construct pre-foams embedded in $\mathbb{R} \times[-1,1] \times[-1,1]$ with vertical boundary components. These vertical boundary components should be the boundary of the webs at the bottom/top times $[-1,1]$. We consider such pre-foams modulo isotopies in $\mathbb{R} \times[-1,1] \times[-1,1]$ which fix the horizontal boundary as well as the vertical boundary, and the condition that generic slices are webs.

We call pre-foam parts ordinary, if they do not contain singular seams or phantom facets, and we call pre-foam parts ghostly, if they only contain phantom facets.

Example 2.6. Pre-foams can be seen as singular surfaces (with oriented, singular seams) in $\mathbb{R} \times[-1,1] \times[-1,1]$ such that the bottom boundary and the top boundary are webs with facets colored as follows:

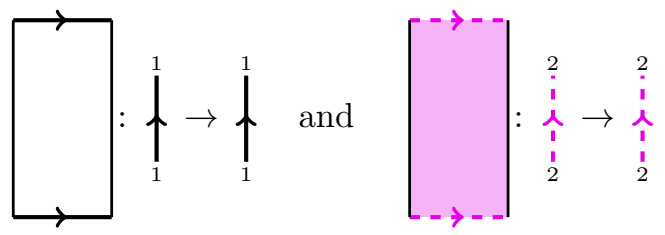

The leftmost facet is an ordinary facet. Whereas the rightmost facet is a phantom facet, and the reader might think of it as "non-existing" (similar to a phantom edge) - they only encode signs. The singularities of $f$ are all locally of the following form (where the other orientations of the seams are also allowed)

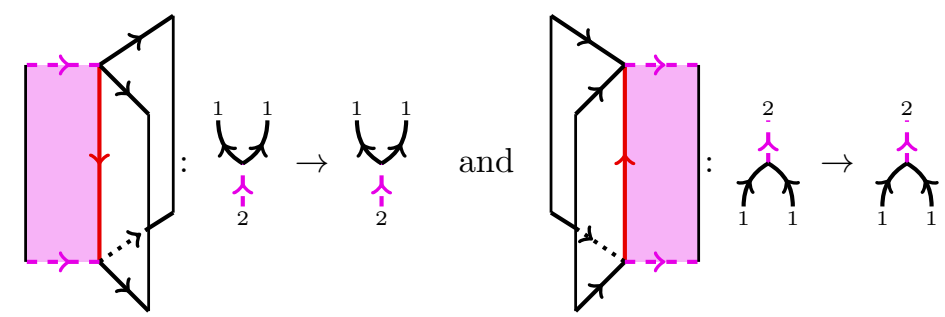

(Note that we consider webs in a "monoidal" way. Thus, we do not have to relate the orientations of facets/seam to the orientations of webs as e.g. in [2, Section 1].) Such pre-foams can carry dots that freely move around its facets:

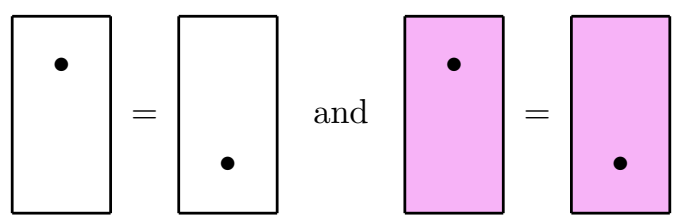

Remark 2.7. Pre-foams are considered modulo boundary preserving isotopies that do preserve the condition that each generic slice is a web. These isotopies form a finite list: isotopies coming from the two cobordism theories associated to the two different types of facets (see for example [22, Section 1.4]) and isotopies coming from isotopies of the singular seams seen as tangles in $\mathbb{R}^{2} \times[-1,1]$.

To work with the 2-category of foams it will be enough (for our purposes) to consider its image under a certain (singular) TQFT functor defined by Blanchet 2]. Recall that equivalence classes of TQFTs for surfaces (i.e. a monoidal functor $\mathcal{Z}$ 
from the category of two-dimensional cobordisms to the category of $\mathbb{K}$-vector spaces) are in one-to-one correspondence with isomorphism classes of (finite-dimensional, associative) commutative Frobenius algebras. The reader unfamiliar with these notions might want to consult Kock's book 22 for a detailed account. Given a Frobenius algebra $\mathcal{A}$ corresponding to a TQFT $\mathcal{Z}_{\mathcal{A}}$, then the association is as follows. To a disjoint union of $m$ circles one associates the $m$-fold tensor product $\mathcal{A}^{\otimes m}$. To a cobordism $\Sigma$ with distinguished incoming and outgoing boundary components consisting of, let us say, $m$ and $m^{\prime}$ circles, we assign a $\mathbb{K}$-linear map from $\mathcal{A}^{\otimes m}$ to $\mathcal{A}^{\otimes m^{\prime}}$. Hereby the usual cup/cap respectively pants cobordisms correspond to the unit, counit, multiplication and comultiplication maps. These are the basic pieces of every cobordism. Then the TQFT assigns to $\Sigma$ a $\mathbb{K}$-linear map

$$
\mathcal{Z}_{\mathcal{A}}(\Sigma): \mathcal{A}^{\otimes m} \rightarrow \mathcal{A}^{\otimes m^{\prime}}
$$

which is obtained by decomposing $\Sigma$ into basic pieces.

The commutative Frobenius algebras we need are

$$
\mathcal{A}_{\mathrm{o}}=\mathbb{K}[X] /\left(X^{2}\right), \quad \mathcal{A}_{\mathrm{p}}=\mathbb{K}
$$

with induced multiplications, counits $\varepsilon_{\mathrm{o}, \mathrm{p}}(\cdot)$ and comultiplications $\Delta_{\mathrm{o}, \mathrm{p}}(\cdot)$ given via

$$
\begin{gathered}
\varepsilon_{\mathrm{o}}(1)=0, \quad \varepsilon_{\mathrm{o}}(X)=1, \quad \varepsilon_{\mathrm{p}}(1)=-1, \\
\Delta_{\mathrm{o}}(1)=1 \otimes X+X \otimes 1, \quad \Delta_{\mathrm{o}}(X)=X \otimes X, \quad \Delta_{\mathrm{p}}(1)=-1 \otimes 1 .
\end{gathered}
$$

Thus, we have the traces

(5) $\operatorname{tr}_{\mathrm{o}}(1 \otimes 1)=\operatorname{tr}_{\mathrm{o}}(X \otimes X)=0, \operatorname{tr}_{\mathrm{o}}(1 \otimes X)=\operatorname{tr}_{\mathrm{o}}(X \otimes 1)=1, \operatorname{tr}_{\mathrm{p}}(1 \otimes 1)=-1$.

We associate the Frobenius algebra $\mathcal{A}_{\mathrm{o}}$ to the ordinary parts, and the Frobenius algebra $\mathcal{A}_{\mathrm{p}}$ to the phantom parts of a pre-foam $f$ using the usual notion of a TQFT functor, but extended to surfaces marked with dots via multiplication by $X$ or -1 :

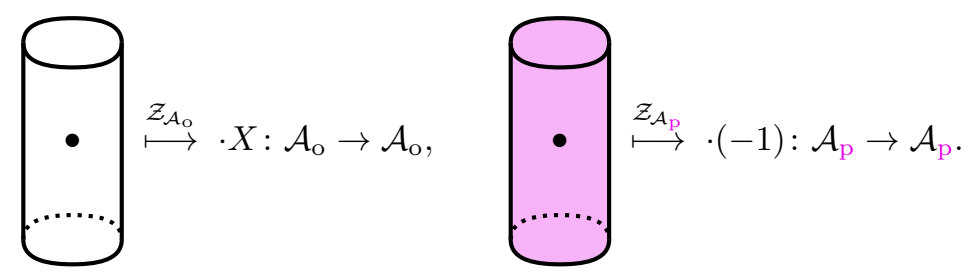

(Note that pre-foams without singular seams and markers are surfaces in the usual sense.) By the universal construction given in [3], it is no problem to extend the usual construction of TQFTs to the marked setup using (6). We leave the details to the reader.

Example 2.8. If we view a $\mathbb{K}$-linear map $\phi: \mathbb{K} \rightarrow \mathcal{A}_{\mathrm{o}, \mathrm{p}}^{\otimes m}$ as $\phi(1) \in \mathcal{A}_{\mathrm{o}, \mathrm{p}}^{\otimes m}$, then

$$
\Longrightarrow \stackrel{\mathcal{Z}_{\mathcal{A}_{\mathrm{o}}}}{\longmapsto} 1 \in \mathcal{A}_{\mathrm{o}}, \quad \longrightarrow \stackrel{\mathcal{Z}_{\mathcal{A}_{\mathrm{o}}}}{\longmapsto} X \in \mathcal{A}_{\mathrm{o}}, \quad \longrightarrow \stackrel{\mathcal{Z}_{\mathcal{A}_{\mathrm{p}}}}{\longmapsto} 1 \in \mathcal{A}_{\mathrm{p}} .
$$

Here we have from left to right $\iota_{\mathrm{o}},(\cdot X) \circ \iota_{\mathrm{o}}$ and $\iota_{\mathrm{p}}$ as maps. These are sometimes called (marked) units. The counits $\varepsilon_{\mathrm{o}, \mathrm{p}}$ are obtained by flipping the pictures.

Note that the values of the non-closed surfaces can be determined by closing them in all possible ways using (7) and its dual. Here and throughout, we say for short that a relation $a=b$ (of formal $\mathbb{K}$-linear combinations of marked surfaces) lies in the kernel of a TQFT functor $\mathcal{Z}$, if $\mathcal{Z}(a)=\mathcal{Z}(b)$ as $\mathbb{K}$-linear maps. (Similarly later on for singular TQFT functors as defined below.) 
Lemma 2.9. The following ordinary sphere relations, the ghostly sphere relation and the cyclotomic relations

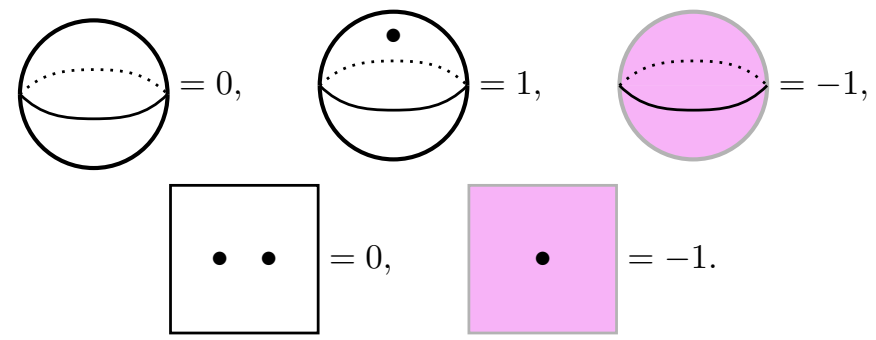

as well as the following ordinary and ghostly neck cutting relations

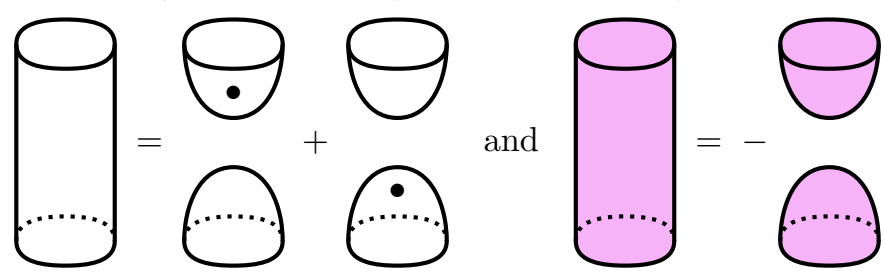

are in the kernel of $\mathcal{T}_{\mathcal{A}_{\mathrm{o}}}$ (ordinary) respectively of $\mathcal{T}_{\mathcal{A}_{\mathrm{p}}}$ (ghostly).

Proof. Via direct calculation. For instance, the ordinary respectively ghostly neck cutting relations decompose the identity map as id $=(\cdot X) \circ \iota_{\mathrm{o}} \circ \varepsilon_{\mathrm{o}}+\iota_{\mathrm{o}} \circ \varepsilon_{\mathrm{o}} \circ(\cdot X)$ respectively id $=-\iota_{\mathrm{p}} \circ \varepsilon_{\mathrm{p}}$ with units $\iota_{\mathrm{o}, \mathrm{p}}$ and counits $\varepsilon_{\mathrm{o}, \mathrm{p}}$ as in (7).

The neck cutting relations 10 give a topological interpretation of a dot as a shorthand notation for ( $\frac{1}{2}$-times) a handle, see also [1, (4)].

2.2. Blanchet's singular TQFT construction. The following definition follows the construction given by Blanchet, see [2, Subsection 1.5].

We want to construct a monoidal functor $\mathcal{T}$ on the category whose objects are webs with values in finite-dimensional $\mathbb{K}$-vector spaces. To this end, let $p \mathbf{F}$ denote the category whose objects are webs and whose morphisms are pre-foams (composition is gluing of pre-foams). We view $p \mathbf{F}$ as a monoidal category by juxtaposition of webs and pre-foams. Moreover, we define for $a, b, c, d \in \mathbb{K}$ two maps:

$$
\begin{gathered}
\alpha_{\mathcal{A}_{\mathrm{o}}}: \mathcal{A}_{\mathrm{o}} \otimes \mathcal{A}_{\mathrm{o}} \rightarrow \mathcal{A}_{\mathrm{o}},(a+b X) \otimes(c+d X) \mapsto(a+b X)(c-d X), \\
\alpha_{\mathcal{A}_{\mathrm{p}}}: \mathcal{A}_{\mathrm{p}} \rightarrow \mathcal{A}_{\mathrm{o}}, 1 \mapsto 1 .
\end{gathered}
$$

Definition 2.10. Let $\mathcal{Z}_{\mathcal{A}_{\mathrm{o}}}$ and $\mathcal{Z}_{\mathcal{A}_{\mathrm{p}}}$ denote the TQFTs associated to $\mathcal{A}_{\mathrm{o}}$ and $\mathcal{A}_{\mathrm{p}}$ from (4). Given a closed pre-foam $f_{c}$, let $\dot{f}_{c}=f_{\mathrm{o}} \dot{\cup} f_{\mathrm{p}}$ be the pre-foam obtained by cutting $f_{c}$ along the singular seams (of which we assume to have $m$ in total). Here $f_{\mathrm{o}}$ is the surface which in $f_{c}$ is attached to the ordinary parts and $f_{\mathrm{p}}$ is the surface which in $f_{c}$ is attached to phantom parts. Note that the boundary of $f_{\mathrm{o}}$ splits into $\sigma_{i}^{+}$and $\sigma_{i}^{-}$for each $i \in\{1, \ldots, m\}$. Which one is which depends on the orientation of the singular seam: use the right hand rule with the index finger pointing in the direction of the singular seam and the middle finger pointing in direction of the attached phantom facet, then the thumb points in direction of $\sigma_{i}^{+}$. In contrast, $f_{\mathrm{p}}$ has only boundary components $\sigma_{i}$ for each $i \in\{1, \ldots, m\}$. Now

$$
\mathcal{Z}_{\mathcal{A}_{\mathrm{o}}}\left(f_{\mathrm{o}}\right) \in \bigotimes_{i=1}^{m}\left(\mathcal{Z}_{\mathcal{A}_{\mathrm{o}}}\left(\sigma_{i}^{+}\right) \otimes \mathcal{Z}_{\mathcal{A}_{\mathrm{o}}}\left(\sigma_{i}^{-}\right)\right), \quad \mathcal{Z}_{\mathcal{A}_{\mathrm{p}}}\left(f_{\mathrm{p}}\right) \in \bigotimes_{i=1}^{m} \mathcal{Z}_{\mathcal{A}_{\mathrm{p}}}\left(\sigma_{i}\right)
$$

Let $\operatorname{tr}_{\mathrm{o}}: \mathcal{A}_{\mathrm{o}} \rightarrow \mathbb{K}$ be as in (5), and let $\alpha_{\mathcal{A}_{\mathrm{o}}}, \alpha_{\mathcal{A}_{\mathrm{p}}}$ be as in 11]. Then we set

$$
\mathcal{T}\left(f_{c}\right)=\left(\operatorname{tr}_{\mathrm{o}}\right)^{\otimes 2 m}\left(\alpha_{\mathcal{A}_{\mathrm{o}}}^{\otimes m}\left(\mathcal{Z}_{\mathcal{A}_{\mathrm{o}}}\left(f_{\mathrm{o}}\right)\right) \otimes \alpha_{\mathcal{A}_{\mathrm{p}}}^{\otimes m}\left(\mathcal{Z}_{\mathcal{A}_{\mathrm{p}}}\left(f_{\mathrm{p}}\right)\right)\right) \in \mathbb{K}^{\otimes 2 m} \cong \mathbb{K}
$$

This gives a well-defined value $\mathcal{T}\left(f_{c}\right) \in \mathbb{K}$ for all closed pre-foams $f_{c}$. 
A crucial insight of Blanchet is that this extends to pre-foams:

Theorem 2.11. The construction from Definition 2.10 can be extended to a monoidal functor $\mathcal{T}: p \mathbf{F} \rightarrow \mathbb{K}$-Vect. (We call such a functor a singular TQFT.)

Proof. This follows from the universal construction from [3]. That is, the only thing one really needs to check for this is that the $\mathbb{K}$-vector spaces constructed via the universal construction are finite-dimensional. This is not a priori clear, but also not hard to show. First observe that the evaluation given above ensures the case for the $\mathbb{K}$-vector space associated to the empty web. By using the relations found below, one can show an analog of Lemma 4.3. which in turn provides recursively that the $\mathbb{K}$-vector space associated to any web is finite-dimensional.

Note the following properties of pre-foams $f$, which follow by construction.

(I) The topological reduction $\hat{f}$ obtained by removing all phantom facets of $f$ is the cobordism theory corresponding to $\mathcal{A}_{\mathrm{o}}$ from (4).

(II) The phantom $\check{f}$ obtained by removing all 1-labeled facets of $f$ is the cobordism theory corresponding to $\mathcal{A}_{\mathrm{p}}$ from (4).

Hence, the relations from (8), (9) and $(10)$ are also in the kernel of the functor $\mathcal{T}$ (for all possible orientations of the boundary webs).

Lemma 2.12. Let $\tilde{f}$ be the pre-foam obtained from a pre-foam $f$ by reversing the orientation of a singular seam. Then $f+\tilde{f}=0$ is in the kernel of $\mathcal{T}$.

Proof. Switching the orientation of a singular seam swaps the attached parts of $\sigma_{1}^{+}$ and $\sigma_{1}^{-}$. In particular, it swaps the two copies of $\mathcal{A}_{\mathrm{o}}$ in the source of $\alpha_{\mathcal{A}_{\mathrm{o}}}$ from (11) and hence, produces an extra sign (we note that the case $b=d=0$ is killed by applying the trace $\varepsilon_{\mathrm{o}}$ in the formula for $\left.\mathcal{T}\left(f_{c}\right)\right)$.

Lemma 2.13. Let $a, b \in \mathbb{Z}_{\geq 0}$. The sphere relations, i.e.

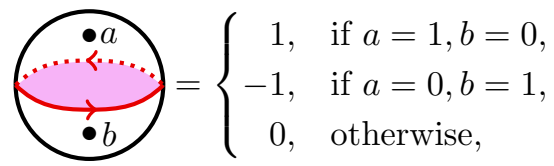

are in the kernel of $\mathcal{T}$. (We call such pre-foams spheres.)

Proof. We prove the case $a=0, b=1$. The others are similar and omitted for brevity. Decompose $f_{c}$ into $(\mathrm{t}=$ thumb, $\mathrm{i}=$ index finger, $\mathrm{m}=$ middle finger $)$
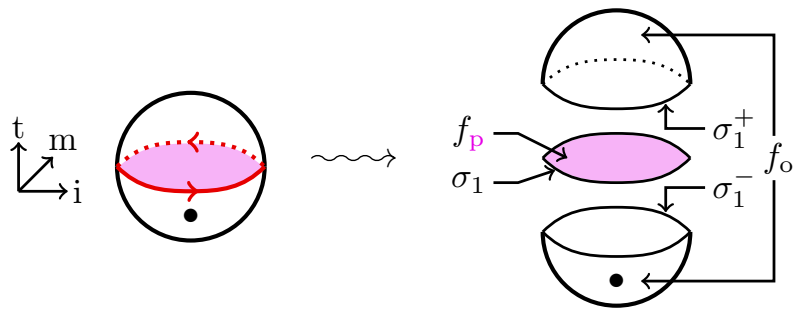

Now, because of the assignment in $(7)$, we have $\mathcal{T}_{\mathcal{A}_{\mathrm{o}}}\left(f_{\mathrm{o}}\right)=1 \otimes X$ and $\mathcal{T}_{\mathcal{A}_{\mathrm{p}}}\left(f_{\mathrm{p}}\right)=1$. Thus, $\alpha_{\mathcal{A}_{\mathrm{o}}}\left(\mathcal{T}_{\mathcal{A}_{\mathrm{o}}}\left(f_{\mathrm{o}}\right)\right)=-X$ and $\alpha_{\mathcal{A}_{\mathrm{p}}}\left(\mathcal{T}_{\mathcal{A}_{\mathrm{p}}}\left(f_{\mathrm{p}}\right)\right)=1$, both considered in $\mathcal{A}_{\mathrm{o}}$. Applying the trace $\operatorname{tr}_{\mathrm{o}}$ to $-X \otimes 1$ gives -1 as in 12 . 
Lemma 2.14. The bubble removals (a "sphere" in a phantom plane; the top dots are meant to be on the front facets and the bottom dots on the back facets)
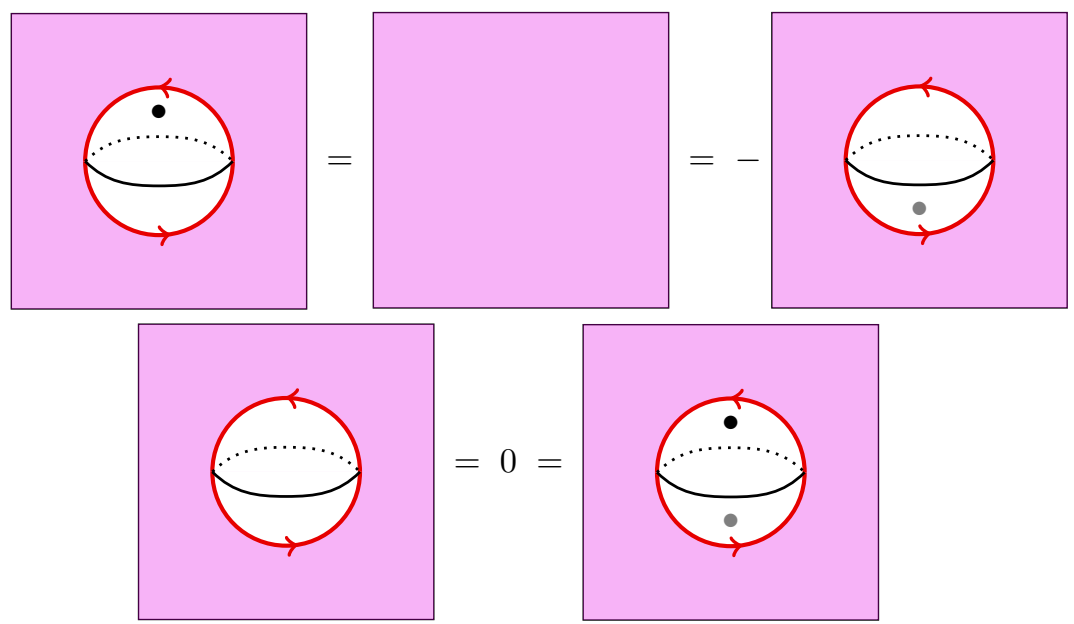

are in the kernel of $\mathcal{T}$. The neck cutting relation
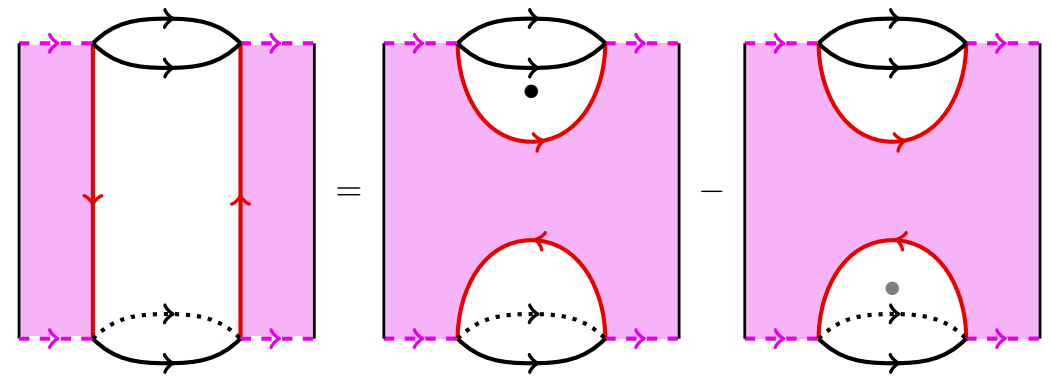

(with top dot on the front facet and bottom dot on the back facet) is also in the kernel of $\mathcal{T}$. Furthermore, the (left) squeezing relation

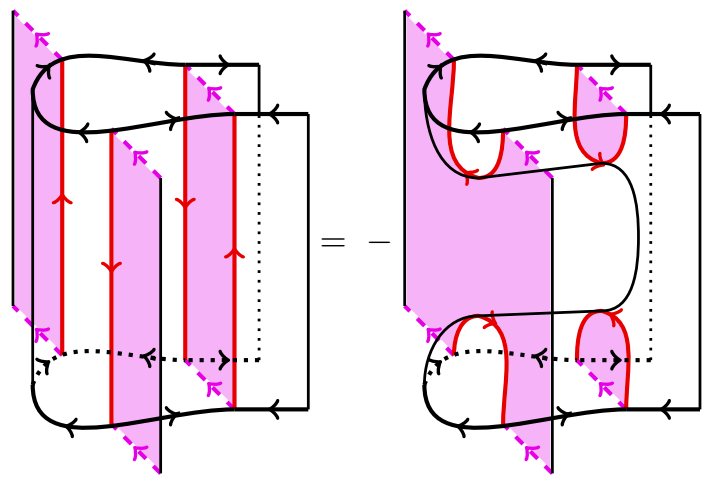

(there is also a similar right squeezing relation) and the dot migrations
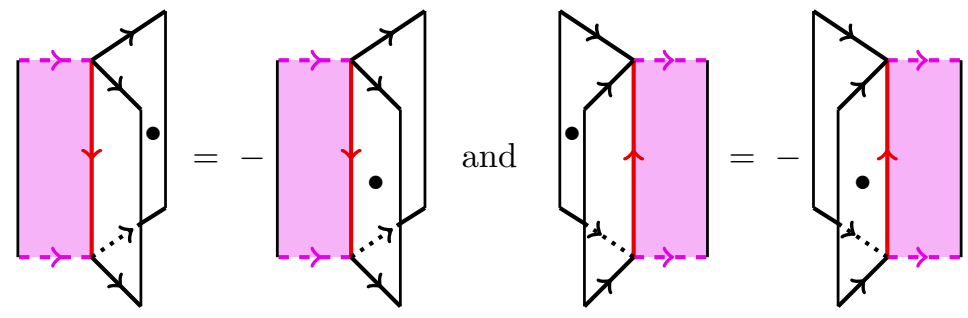
as well as the ordinary-to-phantom neck cutting relations (in the leftmost picture the upper closed circle is an ordinary facet, while the lower closed circle is a phantom facet, and vice versa for the rightmost picture)
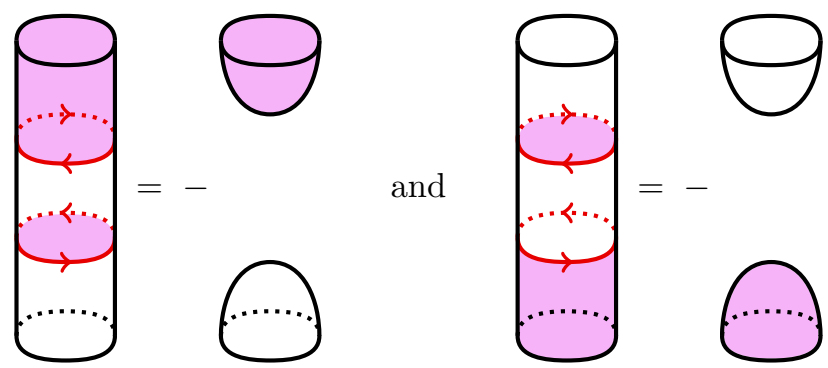

(we have omitted the orientations of the circle-shaped webs) are also in the kernel of $\mathcal{T}$. (We only need these in the following. But there are also similar relations with different orientations of the webs.)

The leftmost situation in 15 is called a cylinder - as all local parts of pre-foams $f$ such that the corresponding part in $\hat{f}$ is a cylinder. Note that the squeezing relation $(16)$ enables us to use the neck cutting 15 on any such cylinders.

Proof. We only prove the left equation in 18 . First note that we have to consider all possible ways to close the non-closed pre-foam on the left-hand and on the right-hand side of the equation. We consider the closing
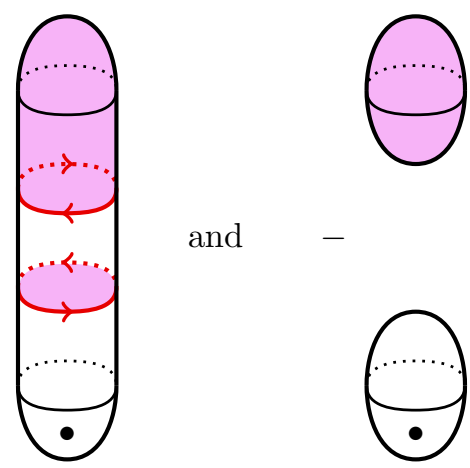

and

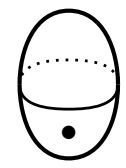

since all other possibilities give zero (as the reader might want to check). By (8), the right-hand closed pre-foams evaluate to $-(1 \cdot(-1))$. Now:
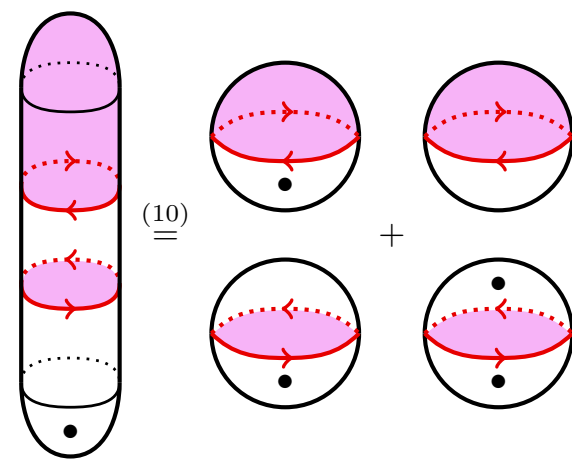

12
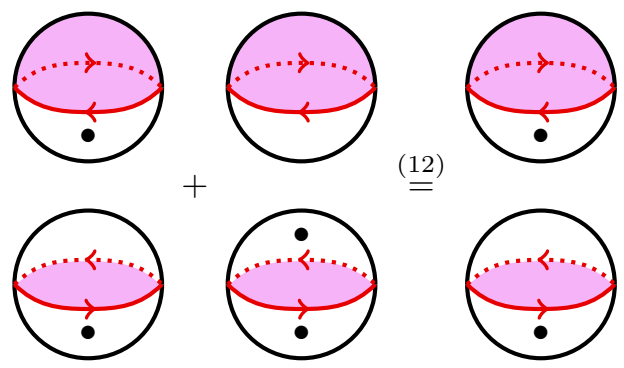

The bottom sphere evaluates to -1 because of 12 . Moreover, performing the same steps as in the proof of Lemma 2.13, we see that the top sphere also evaluates to -1 . Thus, the left-hand and the right-hand side evaluate to the same value. This shows that the first equation in $(18)$ is in the kernel of $\mathcal{T}$. The other relations are verified similarly, see also [2, Lemma 1.3] or [24, Subsection 3.1]. 
If we define a grading on the TQFT-modules by setting $\operatorname{deg}(1)=-1$ and $\operatorname{deg}(X)=1$, then the TQFT $\mathcal{Z}_{\mathcal{A}_{\mathrm{o}}}$ respects the grading, where the degree of a cobordism $\Sigma$ is given by $\operatorname{deg}(\Sigma)=-\chi(\Sigma)+2 \cdot$ dots. Here $\chi(\Sigma)$ is the topological Euler characteristic of $\Sigma$, that is, the number of vertices minus the number of edges plus the number of faces of $\Sigma$ seen as a CW complex, and "dots" is the total number of dots. Additionally, we can see the TQFT $\mathcal{Z}_{\mathcal{A}_{\mathrm{p}}}$ as being trivially graded. Motivated by this we define the following.

Definition 2.15. Given a pre-foam $f$, we define its degree

$$
\operatorname{deg}(f)=-\chi(\hat{f})+2 \cdot \operatorname{dots}+\frac{1}{2} \text { vbound, }
$$

where vbound is the total number of vertical boundary components. If $\hat{f}$ is the empty cobordism, then, by convention, $\chi(\hat{f})=0$.

Example 2.16. For example,
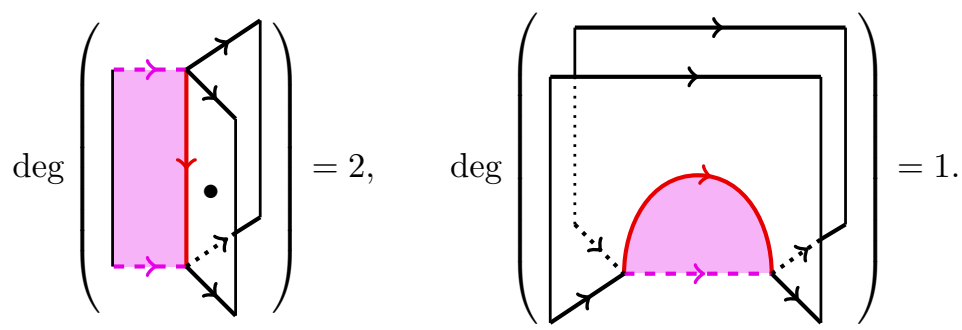

The leftmost pre-foam is called a dotted cup (the name will become clear in Lemma 2.27), while the rightmost pre-foam is called a saddle (there are also saddles obtained by flipping the picture upside down). Furthermore, we have

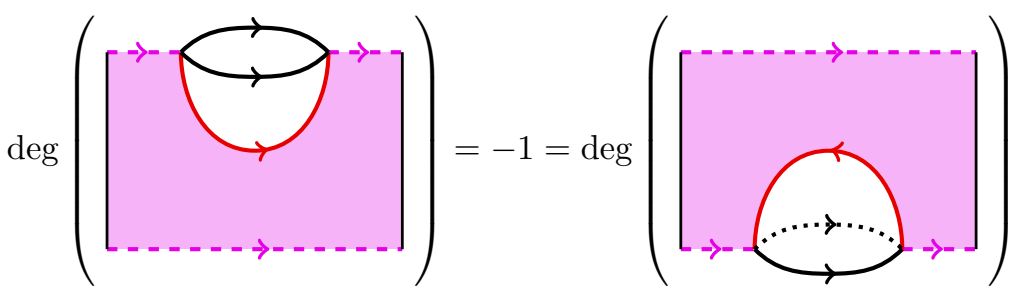

for the pre-foams called cup respectively cap.

The 2-category we like to study is cooked up from $\mathcal{T}$ as follows.

Definition 2.17. Let $\overline{\mathfrak{F}}$ be the $\mathbb{K}$-linear 2-category given by:

- The objects are all $\vec{k} \in \overline{\mathrm{b}}$.

- The morphisms spaces $\operatorname{Hom}_{\overline{\mathfrak{F}}}(\vec{k}, \vec{l})$ are as in Definition 2.1

- The 2-morphisms spaces $2 \operatorname{Hom}_{\overline{\mathfrak{F}}}(u, v)$ for two webs $u, v$ is the $\mathbb{K}$-linear span of all pre-foams with bottom boundary $u$ and top boundary $v$.

- Vertical compositions $g \circ f$ of pre-foams by stacking $g$ on top of $f$, horizontal composition $g \otimes f$ by putting $g$ to the right of $f$ (whenever those operations make sense).

- We take everything modulo the relations from (8), (9) and $(10)$, as well as the relations found in Lemmas 2.12, 2.13 and 2.14 .

Since the relations are degree preserving, $\overline{\mathfrak{F}}$ is a graded, $\mathbb{K}$-linear 2-category by taking the degree from Definition 2.15. Similarly, the "highest weight" 2-subcategory $\mathfrak{F}$ of $\overline{\mathfrak{F}}$ is the full 2 -subcategory consisting of only webs with upwards pointing edges. (We will only consider $\mathfrak{F}$ in the following.)

We call the 2-morphisms in $\mathfrak{F}$ (or in $\overline{\mathfrak{F}}$ ) foams. Moreover, all notions we had for pre-foams can be adapted to the setting of foams and we do so in the following. 
Note that the objects and the morphisms of $\mathfrak{F}$ can be seen as a $\mathbb{K}(q)$-linear category (by considering the spaces $\operatorname{Hom}_{\mathfrak{F}}(\vec{k}, \vec{l})$ as $\mathbb{K}(q)$-linear vector spaces whose basis are the webs in $\left.\operatorname{Hom}_{\mathfrak{F}}(\vec{k}, \vec{l})\right)$ which we denote by Web.

2.3. An action of the quantum group $\dot{\mathrm{U}}_{q}\left(\mathfrak{g l}_{\infty}\right)$. We denote by $\dot{\mathrm{U}}_{q}\left(\mathfrak{g l}_{\infty}\right)$ the $\mathbb{K}(q)$-linear category whose objects are given by $\vec{k} \in \mathbb{b} \mathbb{1}$ and whose morphisms are generated by pairwise orthogonal idempotents $\mathbf{1}_{\vec{k}}$, and by $E_{i}^{(r)} \mathbf{1}_{\vec{k}}$ and $F_{i}^{(r)} \mathbf{1}_{\vec{k}}$ for $\vec{k} \in \mathfrak{b l}, i \in \mathbb{Z}$ and $r \in \mathbb{Z}_{>0}$ (the generators $E_{i}^{(r)} \mathbf{1}_{\vec{k}}$ and $F_{i}^{(r)} \mathbf{1}_{\vec{k}}$ are called the divided powers ) modulo some relations which are analogs of the relations in the quantum group $\mathrm{U}_{q}\left(\mathfrak{g l}_{\infty}\right)$ (see [25, Chapter 23]). We note that there exists a unique $\mathbf{1}_{\vec{l}}$ such that $\mathbf{1}_{\vec{l}} E_{i}^{(r)} \mathbf{1}_{\vec{k}} \neq 0$ and $\mathbf{1}_{\vec{l}} F_{i}^{(r)} \mathbf{1}_{\vec{k}} \neq 0$. This enable us to write $\mathbf{1}_{\vec{k}}$ only on one side of any expression. Now, there is a $\mathbb{K}(q)$-linear functor

$$
\Phi_{\text {Howe }}^{\text {Web }}: \dot{\mathrm{U}}_{q}\left(\mathfrak{g l}_{\infty}\right) \rightarrow \text { Web }
$$

given on objects by $\Phi_{\text {Howe }}^{\mathbf{W e b}}(\vec{k})=\vec{k}$, and on morphisms by $\Phi_{\text {Howe }}^{\mathbf{W e b}}\left(\mathbf{1}_{\vec{k}}\right)=\mathbf{1}_{\vec{k}}$ and (where we use a simplified, "rectangular", notation for webs):

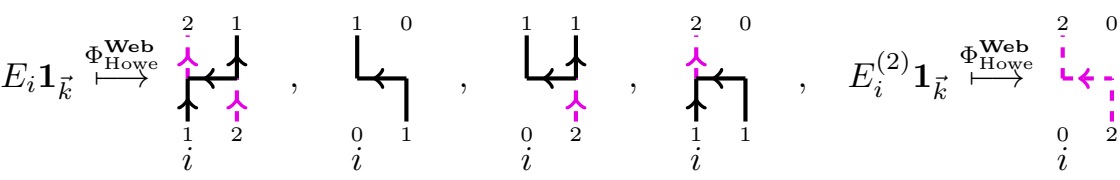

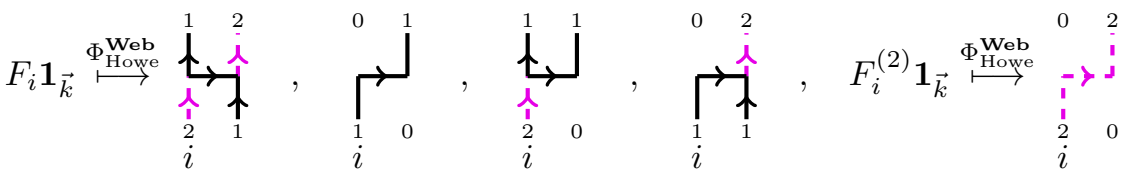

Here the generators $E_{i} \mathbf{1}_{\vec{k}}, F_{i} \mathbf{1}_{\vec{k}}, E_{i}^{(2)} \mathbf{1}_{\vec{k}}$ and $F_{i}^{(2)} \mathbf{1}_{\vec{k}}$ are sent to the local (between strand $i$ and $i+1$ ) pictures above (we have displayed all possibilities depending on $\vec{k}$ at position $k_{i}$ and $\left.k_{i+1}\right)$. Moreover, all higher divided powers $E_{i}^{(r)} \mathbf{1}_{\vec{k}}, F_{i}^{(r)} \mathbf{1}_{\vec{k}}$ for $r>2$ are sent to zero. We call webs that arise as $\Phi_{\text {Howe }}^{\text {Web }}(X)$, for $X$ being any composition of the $\mathbf{1}_{\vec{k}}, E_{i}^{(r)} \mathbf{1}_{\vec{k}}, F_{i}^{(r)} \mathbf{1}_{\vec{k}}$ generators, EF-generated, and, on the other hand, webs $F$-generated, if $X$ is any composition of only $\mathbf{1}_{\vec{k}}, F_{i}^{(r)} \mathbf{1}_{\vec{k}}$ generators (in both cases no coefficients from $\mathbb{K}(q)$ are allowed to occur). For details about the functor $\Phi_{\text {Howe }}^{\mathrm{Web}}$ we refer to [9, Section 5].

Example 2.18. If $\vec{k}=(0,0,1,0,2,0,1,2,0,0)$, then $E_{1} E_{0} E_{-1} E_{2} F_{0} \mathbf{1}_{\vec{k}}$ is sent to

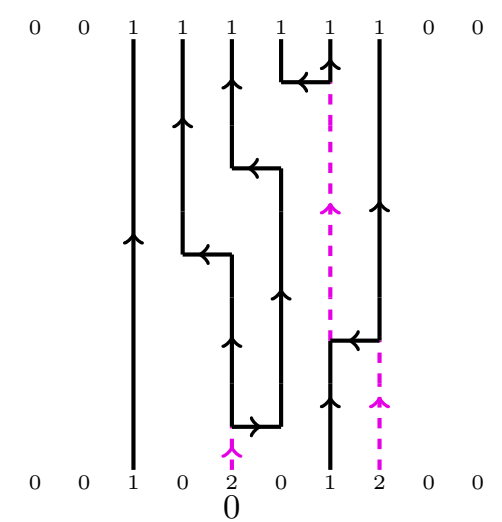

Here the first entry 2 is assumed to be at position 0 . 
2.4. $\mathfrak{g l}_{2}$-web algebras. Now we define the following "algebraic" version of $\mathfrak{F}$. To this end, let $\ell \in \mathbb{Z}_{\geq 0}$ and let $\omega_{\ell}=(1, \ldots, 1,0, \ldots, 0)$ with $\ell$ numbers equal to 1 . Fix $\vec{k}$ and let $\operatorname{Cup}(\vec{k})=\operatorname{Hom}_{\mathfrak{F}}\left(2 \omega_{\ell}, \vec{k}\right)$ and $\operatorname{Cap}(\vec{k})=\operatorname{Hom}_{\mathfrak{F}}\left(\vec{k}, 2 \omega_{\ell}\right) .(\operatorname{Thus}, \operatorname{Cup}(\vec{k})=\emptyset$ and $\operatorname{Cap}(\vec{k})=\emptyset$ if $\sum_{i \in \mathbb{Z}} k_{i} \neq 2 \ell$.) Elements of these are called cup webs respectively cap webs. For diagrams in the multiplication process of $\mathfrak{W}$ described below, we need cup-ray webs as well, i.e. elements of $\operatorname{CupRay}(\vec{k})=\operatorname{Hom}_{\mathfrak{F}}\left(\omega_{\ell+\ell^{\prime}}+\omega_{\ell}, \vec{k}\right)$ for $\vec{k} \in \mathbb{b} \mathbb{1}$ (and similarly defined cap-ray webs).

Definition 2.19. Let $u, v \in \operatorname{Cup}(\vec{k}), \vec{k} \in \mathfrak{b l}$. We denote by ${ }_{u}\left(\mathfrak{W}_{\vec{k}}^{\natural}\right)_{v}$ the space $2 \operatorname{Hom}_{\mathfrak{F}}(u, v)$. The web algebra $\mathfrak{W}_{\vec{k}}^{\natural}$ for $\vec{k} \in \mathbb{b} \mathbb{1}$ and the (full) web algebra $\mathfrak{W}^{\natural}$ are the graded $\mathbb{K}$-vector spaces

$$
\mathfrak{W}_{\vec{k}}^{\natural}=\bigoplus_{u, v \in \operatorname{Cup}(\vec{k})}{ }_{u}\left(\mathfrak{W}_{\vec{k}}^{\natural}\right)_{v}, \quad \mathfrak{W}^{\natural}=\bigoplus_{\vec{k} \in \mathfrak{B} \mathbb{1}} \mathfrak{W}_{\vec{k}}^{\natural},
$$

whose grading is induced by the grading in $\mathfrak{F}$. We consider these as graded algebras with multiplication given by composition in $\mathfrak{F}$.

Remark 2.20. Note that $\mathfrak{W J}_{\vec{k}}^{\natural}$ is defined via composition of foams and thus, forms a graded, associative, unital algebra. Similarly for (the locally unital) algebra $\mathfrak{W}^{\natural}$.

Remark 2.21. Although new in this form, the algebras from Definition 2.19 are of course inspired by Khovanov's original arc algebras from [18. Consequently, we obtain that $\mathfrak{W}_{\vec{k}}$ is a graded Frobenius algebra (by copying [27, Theorem 3.9]).

Definition 2.22. Denote by $\mathfrak{b} \mathbb{1}^{\diamond} \subset \mathbb{b} \mathbb{1}$ the set of all $\vec{k} \in \mathbb{b} \mathbb{l}$ which have an even number of entries 1 . We call elements of $\mathrm{b}^{\diamond}$ balanced.

Remark 2.23. Clearly there are no cups respectively caps if $\vec{k} \in \mathfrak{b} \mathbb{1}-\mathfrak{b} \mathbb{1}^{\diamond}$. Hence, $\mathfrak{W}_{\vec{k}}^{\natural}=0$ iff $\vec{k} \in \mathfrak{b} \mathbb{\mathbb { 1 }}-\mathfrak{b} \mathbb{1}^{\diamond}$ or $\vec{k}=\emptyset$. Consequently, we restrict ourselves to balanced $\vec{k}$ in what follows. We note that the full set $\mathfrak{b} \mathbb{1}$ would be needed if one wants to study generalized web algebras in the sense of [4] or [6].

There is an alternative way to define the web algebras which is the one we will use later on. Thus, we make the following definition (and show below that it agrees with the one from Definition 2.19. We denote by ${ }^{*}$ the involution on webs which flips the diagrams upside down and reverses their orientations.

Definition 2.24. Let $u, v \in \operatorname{Cup}(\vec{k}), \vec{k} \in \mathfrak{b}^{\diamond}$. We denote by ${ }_{u}\left(\mathfrak{W}_{\vec{k}}\right)_{v}$ the space $2 \operatorname{Hom}_{\mathfrak{F}}\left(\mathbf{1}_{2 \omega_{\ell}}, u v^{*}\right)\{d(\vec{k})\}$, where $d(\vec{k})=\ell-\sum_{i \in \mathbb{Z}} k_{i}\left(k_{i}-1\right)$. The web algebra $\mathfrak{W}_{\vec{k}}$ for $\vec{k} \in \mathbb{b} \mathbb{1}^{\diamond}$ and the (full) web algebra $\mathfrak{W}$ are the graded $\mathbb{K}$-vector spaces

$$
\mathfrak{W}_{\vec{k}}=\bigoplus_{u, v \in \operatorname{Cup}(\vec{k})}\left(\mathfrak{W}_{\vec{k}}\right)_{v}, \quad \mathfrak{W}=\bigoplus_{\vec{k} \in \mathfrak{b}^{\triangleright}} \mathfrak{W}_{\vec{k}} .
$$

We consider these as graded algebras with multiplication

$$
\text { Mult : } \mathfrak{W}_{\vec{k}} \otimes \mathfrak{W}_{\vec{k}} \rightarrow \mathfrak{W}_{\vec{k}}, f \otimes g \mapsto \operatorname{Mult}(f, g)=f g
$$

using multiplication foams as follows. To multiply $f \in{ }_{u}\left(\mathfrak{W}_{\vec{k}}\right)_{v}$ with $g \in{ }_{v}\left(\mathfrak{W}_{\vec{k}}\right)_{w}$ stack the diagram $\tilde{v} w^{*}$ on top of $u v^{*}$ and obtain $u v^{*} \tilde{v} w^{*}$. Then $f g=0$ if $v \neq \tilde{v}$. Otherwise, pick the leftmost cup-cap pair indicated in the picture to the left below and perform a "surgery"
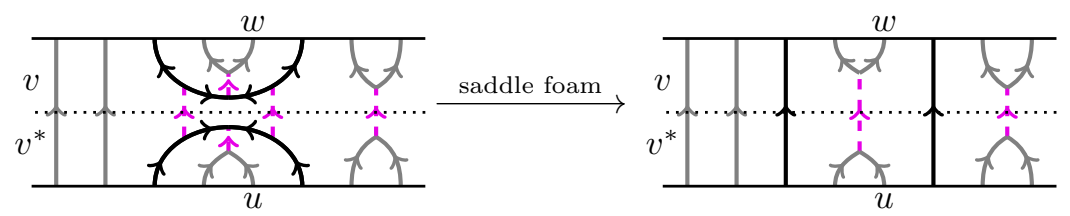
where the saddle foam is locally of the following form (and the identity elsewhere)

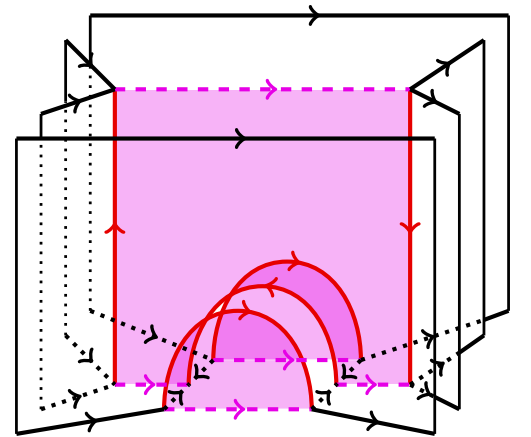

This foam should be read as follows: start with $f \in 2 \operatorname{Hom}_{\mathfrak{F}}\left(\mathbf{1}_{2 \omega_{\ell}}, u v^{*} v w\right)$ and stack on top of it a foam which is the identity at the bottom ( $u$ part) and top ( $w$ part) of the web and the saddle in between. Repeat until no cup-cap pair as above remains. This gives inductively rise to a multiplication foam (after the last surgery step we collapse the webs and foams). Compare also to [27, Definition 3.3].

Note that each intermediate step in the multiplication from 20 is a web of the form $v^{*} v$ with $v \in \operatorname{CapRay}(\vec{k})$ and the multiplication foam is zero or a foam in $\operatorname{Hom}_{\mathfrak{F}}\left(u v^{*} v w^{*}, u w^{*}\right)$ (and thus, locally a foam in $\operatorname{Hom}_{\mathfrak{F}}\left(v^{*} v, \mathbf{1}_{\vec{k}}\right)$ ). As a convention, we consider $u \in \operatorname{Cup}(\vec{k})$ as a web in $\mathbb{R} \times[-1,0], v^{*} \in \operatorname{Cap}(\vec{k})$ as a web in $\mathbb{R} \times[0,1]$ such that $\vec{k} \in \mathbb{R} \times\{0\}$ whenever we use this viewpoint on $\mathfrak{W}$. Similarly for cup-ray and cap-ray webs.

Lemma 2.25. The multiplication is degree preserving.

Proof. The saddles as in (21) are always of degree 1. These have two boundary components labeled 1 . Thus, the degree of all saddles within the multiplication procedure and the shift by $d(\vec{k})$ sum up to zero.

Example 2.26. An easy example illustrating the multiplication is
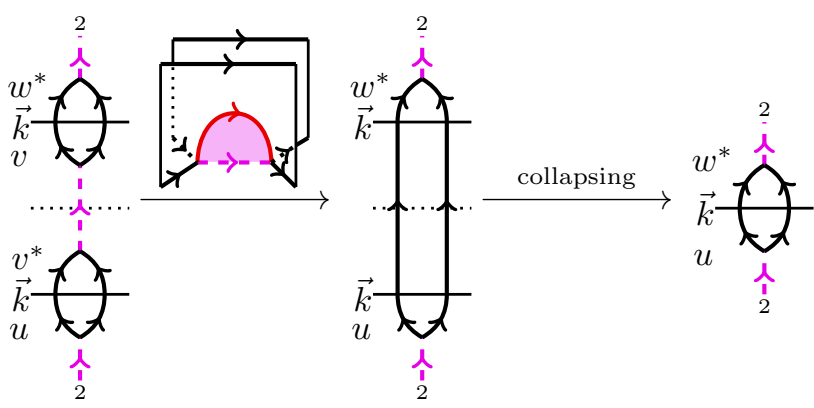

where the reader should think about any foam $f: \mathbf{1}_{2 \omega_{1}} \rightarrow u v^{*} v w^{*}$ sitting underneath. The saddle is of degree 1 and thus, taking the shift $d(\vec{k})$ into account for $\vec{k}=(1,1)$, the multiplication foam is of degree zero.

The following shows that Definitions 2.19 and 2.24 agree.

Lemma 2.27. We have $\mathfrak{W}_{\vec{k}}^{\natural} \cong \mathfrak{W}_{\vec{k}}$ and $\mathfrak{W}^{\natural} \cong \mathfrak{W}$ as graded algebras.

Proof. Recall that the multiplication in $\mathfrak{W}_{\vec{k}}^{\natural}$ is composition, while the multiplication in $\mathfrak{W}_{\vec{k}}$ is given by multiplication foams. Thus, for the former we take foams $f \in 2 \operatorname{Hom}_{\mathfrak{F}}(u, v)$ and $g \in 2 \operatorname{Hom}_{\mathfrak{F}}(v, w)$ and obtain a foam $g \circ f \in 2 \operatorname{Hom}_{\mathfrak{F}}(u, w)$, while for the latter we take foams $f \in 2 \operatorname{Hom}_{\mathfrak{F}}\left(\mathbf{1}_{2 \omega_{\ell}}, u v^{*}\right)$ and $g \in 2 \operatorname{Hom}_{\mathfrak{F}}\left(\mathbf{1}_{2 \omega_{\ell}}, v w^{*}\right)$ 
and obtain a foam $f g \in 2 \operatorname{Hom}_{\mathfrak{F}}\left(\mathbf{1}_{2 \omega_{\ell}}, u w^{*}\right)$ (in case the multiplication is non-zero). Now, the following "clapping of pictures" (as indicated by the arrows)
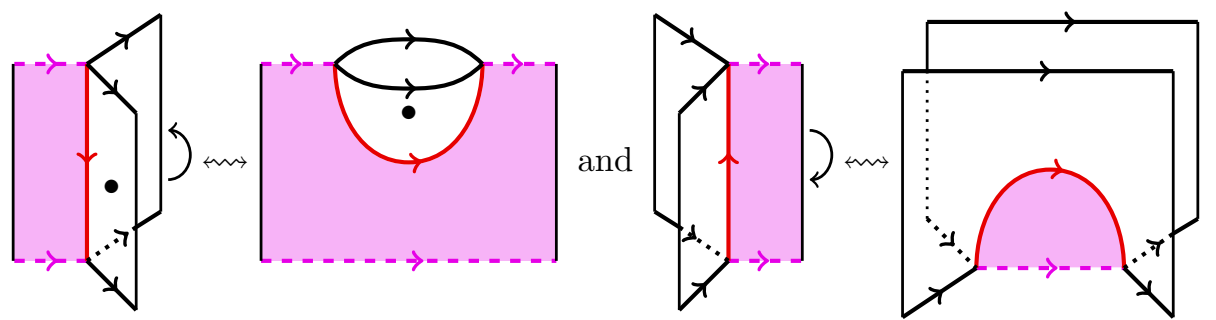

induces isomorphisms of $\mathbb{K}$-vector spaces

$$
\begin{aligned}
2 \operatorname{Hom}_{\mathfrak{F}}(u, w) & \cong 2 \operatorname{Hom}_{\mathfrak{F}}\left(\mathbf{1}_{2 \omega_{\ell}}, u w^{*}\right)\{d(\vec{k})\}, \\
2 \operatorname{Hom}_{\mathfrak{F}}(v, v) & \cong 2 \operatorname{Hom}_{\mathfrak{F}}\left(v^{*} v, \mathbf{1}_{2 \omega_{\ell}}\right)\{d(\vec{k})\} .
\end{aligned}
$$

These are isomorphisms of graded $\mathbb{K}$-vector spaces since the shift by $d(\vec{k})$ encodes the vertical boundary components which are "lost" by the "clapping". Moreover, as indicated in the rightmost picture above, the multiplications in $\mathfrak{W}_{\vec{k}}^{\natural}$ and $\mathfrak{W}_{\vec{k}}$ are identified under this "clapping procedure". This shows the isomorphism of graded $\mathbb{K}$-algebras. For more details the reader might also consult [27, Lemma 3.7].

As a direct consequence of Remark 2.20 and Lemma 2.27 we obtain in particular the associativity of $\mathfrak{W}_{\vec{k}}$ :

Corollary 2.28. The map Mult: $\mathfrak{W}_{\vec{k}} \otimes \mathfrak{W}_{\vec{k}} \rightarrow \mathfrak{W}_{\vec{k}}$ from Definition 2.24 is independent of the order in which the surgeries are performed. This turns $\mathfrak{W}_{\vec{k}}$ into a graded, associative, unital algebra. Similar for (the locally unital) algebra $\mathfrak{W}$.

2.5. Web bimodules. We still consider only balanced $\vec{k}, \vec{l} \in \mathbb{b} \mathbb{1}^{\diamond}$ in this subsection.

Definition 2.29. Given any web $u \in \operatorname{Hom}_{\mathfrak{F}}(\vec{k}, \vec{l})$ (with boundaries $\vec{k}$ and $\vec{l}$ summing up to $2 \ell$ ), we consider the $\mathfrak{W}$-bimodule

$$
\mathfrak{W}(u)=\bigoplus_{\substack{v \in \operatorname{Cup}(\vec{k}), w \in \operatorname{Cup}(\vec{l})}} 2 \operatorname{Hom}_{\mathfrak{F}}\left(\mathbf{1}_{2 \omega_{\ell}}, v w^{*}\right)
$$

with left (bottom) and right (top) action of $\mathfrak{W}$ as in Definition 2.24. We call such $\mathfrak{W}$-bimodules $\mathfrak{W}(u)$ web bimodules.

Proposition 2.30. Let $u \in \operatorname{Hom}_{\mathfrak{F}}(\vec{k}, \vec{l})$ be a web. Then the left (bottom) action of $\mathfrak{W}_{\vec{k}}$ and the right (top) action of $\mathfrak{W}_{\vec{l}}$ on $\mathfrak{W}(u)$ are well-defined and commute. Hence, $\mathfrak{W}(u)$ is a $\mathfrak{W}_{\vec{k}}-\mathfrak{W}_{\vec{l}}$-bimodule (and thus, a $\mathfrak{W}$-bimodule).

Proof. Let $u \in \operatorname{Hom}_{\mathfrak{F}}(\vec{k}, \vec{l})$. Then, by construction, the left (bottom) action of $\mathfrak{W}_{\vec{k}}$ and the right (top) action of $\mathfrak{W}_{\vec{l}}$ commute since they are topologically "far apart". Hence, $\mathfrak{W}(u)$ is indeed a $\mathfrak{W}_{\vec{k}}-\mathfrak{W}_{\vec{l}}$-bimodule (and thus, a $\mathfrak{W}$-bimodule).

Note that, given two webs $u, v \in \operatorname{Hom}_{\mathfrak{F}}(\vec{k}, \vec{l})$, then $\mathfrak{W}(u)$ and $\mathfrak{W}(v)$ could be isomorphic even though $u$ and $v$ are different, see for example (35).

Proposition 2.31. The $\mathfrak{W}$-bimodules $\mathfrak{W}(u)$ are graded biprojective $\mathfrak{W}$-bimodules, with finite-dimensional subspaces for all pairs $v, w$.

Proof. Clearly, they are graded, $\mathfrak{W}$-bimodules, but finite-dimensionality is not a priori clear. It follows from the existence of a cup foam basis as in Subsection 4.3 . (More precisely, from Lemma 4.14) They are also biprojective, because they are direct summands of some $\mathfrak{W}_{\vec{k}}$ (of some $\mathfrak{W}_{\vec{l}}$ ) as left (right) modules and for suitable $\vec{k} \in \mathbb{b} \mathbb{D}^{\triangleright}$ (or $\vec{l} \in \mathbb{b} \mathbb{D}^{\triangleright}$ ). See also [27, Proposition 5.11]. 
This proposition motivates the definition of the following 2-category which is one of the main objects that we are going to study.

Definition 2.32. Given $\mathfrak{W}$ as above, let $\mathfrak{W}$-biMod ${ }_{\mathrm{gr}}^{p}$ be the following 2-category:

- Objects are the various $\vec{k} \in \mathbb{b} \mathbb{1}^{\diamond}$.

- Morphisms are finite sums and tensor products (taken over the algebra $\mathfrak{W}$ ) of $\mathfrak{W}$-bimodules $\mathfrak{W}(u)$.

- The composition of $\mathfrak{W}$-bimodules is given by tensoring (over $\mathfrak{W})$.

- 2-morphisms are $\mathfrak{W}$-bimodule homomorphisms.

- The vertical composition of $\mathfrak{W}$-bimodule homomorphisms is the usual composition and the horizontal composition is given by tensoring (over $\mathfrak{W}$ ).

We consider $\mathfrak{W}$-biMod ${ }_{\text {gr }}^{p}$ as a graded 2-category with 2-hom-spaces as in (1).

\section{Blanchet-Khovanov AlgEBras}

In this section we define the Blanchet-Khovanov algebra, following the framework of [18] and 4], but with signs differing at a number of crucial places.

3.1. Combinatorics of arc diagrams. We start with the notion of weights and blocks. These definitions are the same as in [4, Section 2] and, apart from the exact definition of blocks, as in [12, Sections 2 and 3].

Definition 3.1. A (diagrammatical) weight is a sequence $\lambda=\left(\lambda_{i}\right)_{i \in \mathbb{Z}}$ with entries $\lambda_{i} \in\{\circ, \times, \vee, \wedge\}$, such that $\lambda_{i}=\circ$ for $|i| \gg 0$. Two weights $\lambda$ and $\mu$ are said to be equivalent if one can obtain $\mu$ from $\lambda$ by permuting some symbols $\wedge$ and $\vee$ in $\lambda$. The equivalence classes are called blocks. We denote by bl the set of blocks.

To a block we assign a number of invariants.

Definition 3.2. Let $\Lambda \in$ bl be a block. To $\Lambda$ we associate its (well-defined) block sequence $\operatorname{seq}(\Lambda)=\left(\operatorname{seq}(\Lambda)_{i}\right)_{i \in \mathbb{Z}}$ by taking any $\lambda \in \Lambda$ and replacing the symbols $\wedge, \vee$ by $\star$. Moreover, we define $\operatorname{up}(\Lambda)$ respectively $\operatorname{down}(\Lambda)$ to be the total number of $\wedge$ 's respectively $\vee$ 's in $\Lambda$ where we count $\times$ as both, $\wedge$ and $\vee$.

Important for us is the following subset of blocks.

Definition 3.3. A block $\Lambda \in \mathrm{bl}$ is called balanced, if $\mathrm{up}(\Lambda)=\operatorname{down}(\Lambda)$. We denote by $\mathrm{bl}^{\diamond} \subset \mathrm{bl}$ the set of balanced blocks.

Remark 3.4. The Blanchet-Khovanov algebras will only be defined for balanced blocks, while general blocks can be used to define a generalized version of these algebras in the spirit of [4].

A cup diagram $c$ is a finite collection of non-intersecting arcs inside $\mathbb{R} \times[-1,0]$ such that each arc intersects the boundary exactly in its endpoints, and either connecting two distinct points $(i, 0)$ and $(j, 0)$ with $i, j \in \mathbb{Z}$ (called a cup), or connecting one point $(i, 0)$ with $i \in \mathbb{Z}$ with a point on the lower boundary of $\mathbb{R} \times[-1,0]$ (called a ray). Furthermore, each point in the boundary is endpoint of at most one arc. Two cup diagrams are equal if the arcs contained in them connect the same points. Similarly, a cap diagram $d^{*}$ is defined inside $\mathbb{R} \times[0,1]$. By construction, one can reflect a cup diagram $c$ along the axis $\mathbb{R} \times\{0\}$, denote this operation by ${ }^{*}$, to obtain a cap diagram $c^{*}$. Clearly, $\left(c^{*}\right)^{*}=c$.

A cup diagram $c$ (and similarly a cap diagram $d^{*}$ ) is compatible with a block $\Lambda \in \mathrm{bl}$ if $\left\{(i, 0) \mid \operatorname{seq}(\Lambda)_{i}=\star\right\}=(\mathbb{R} \times\{0\}) \cap c$.

We will view a weight $\lambda$ as labeling integral points, called vertices, of the horizontal line $\mathbb{R} \times\{0\}$ inside $\mathbb{R} \times[-1,0]$ and $\mathbb{R} \times[0,1]$, putting the symbol $\lambda_{i}$ at position $(i, 0)$. Together with a cup diagram $c$ this forms a new diagram $c \lambda$. 
Definition 3.5. We say that $c \lambda$ is oriented if:

(I) An arc in $c$ only contains vertices labeled by $\wedge$ or $\vee$.

(II) The two vertices of a cup are labeled by exactly one $\wedge$ and one $\vee$.

(III) Every vertex labeled $\wedge$ or $\vee$ is contained in an arc.

(IV) It is not possible to find $i<j$ such that $\lambda_{i}=\vee, \lambda_{j}=\wedge$, and each are contained in a ray.

In the following, when depicting a composite diagram like $c \lambda$, we will omit the line and only draw the labels obtained from $\lambda$.

Example 3.6. Consider the following diagrams.

(i)

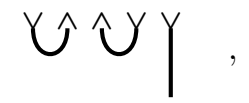

(ii)

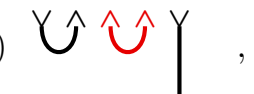

(iii)

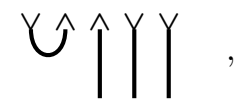

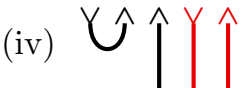

The diagrams (i) and (iii) are oriented. Diagram (ii) is not oriented since condition (II) is violated, while (iv) is not oriented because condition (IV) is not fulfilled.

Similarly, a cap diagram $d^{*}$ together with a weight $\lambda$ forms a diagram $\lambda d^{*}$, which is called oriented if $d \lambda$ is oriented. A cup respectively a cap in such diagrams is called anticlockwise (or clockwise), if its rightmost vertex is labeled $\wedge$ (or $\vee$ ).

Putting a cap diagram $d^{*}$ on top of a cup diagram $c$ such that they are connected to the line $\mathbb{R} \times\{0\}$ at the same points creates a circle diagram, denoted by $c d^{*}$. All connected component of this diagram that do not touch the boundary of $\mathbb{R} \times[-1,1]$ are called circles, all others are called lines. Together with $\lambda \in \Lambda$ such that $c \lambda$ and $\lambda d^{*}$ are oriented this forms an oriented circle diagram $c \lambda d^{*}$.

Definition 3.7. We define the degree of an oriented cup diagram $c \lambda$, of an oriented cap diagram $\lambda d^{*}$ and of an oriented circle diagram $c \lambda d^{*}$ as follows.

$$
\begin{aligned}
\operatorname{deg}(c \lambda) & =\text { number of clockwise cups in } c \lambda, \\
\operatorname{deg}\left(\lambda d^{*}\right) & =\text { number of clockwise caps in } \lambda d^{*}, \\
\operatorname{deg}\left(c \lambda d^{*}\right) & =\operatorname{deg}(c \lambda)+\operatorname{deg}\left(\lambda d^{*}\right) .
\end{aligned}
$$

Note that the degree is always non-negative.

Example 3.8. In Example 3.6 above, diagram (i) has degree 1 (due to one clockwise cup) and diagram (iii) has degree 0.

Finally, we associate to each $\lambda \in \Lambda$ a unique cup diagram, denoted by $\underline{\lambda}$, via:

(I) Connect neighboring pairs $\vee \wedge$ with a cup, ignoring symbols of the type $\circ$ and $\times$ as well as symbols already connected. Repeat this process until there are no more $\vee$ 's to the left of any $\wedge$.

(II) Put a ray under any remaining symbols $\vee$ or $\wedge$.

It is an easy observation that $\underline{\lambda}$ always exists for a fixed $\lambda$. Furthermore, $\lambda$ is the (unique) orientation of $\underline{\lambda}$, such that $\underline{\lambda} \lambda$ has minimal degree. Each cup diagram $c$ is of the form $\lambda$ for $\lambda \in \Lambda$, a block compatible with $c$.

Similarly we can define $\bar{\lambda}=\underline{\lambda}^{*}$, and, as before, in an oriented circle diagram $\underline{\lambda} \nu \bar{\mu}$ a circle $C$ is said to be oriented anticlockwise if the rightmost vertex contained in the circle is $\wedge$ and clockwise in case its $\vee$. Two helpful facts about the degree of oriented circle diagrams are summarized below.

Lemma 3.9. Fix a block $\Lambda$ and $\lambda, \mu, \nu \in \Lambda$.

(a) The contribution to the degree of the arcs contained in a given circle $C$ inside an oriented circle diagram $\underline{\lambda} \nu \bar{\mu}$ is equal to

$$
\operatorname{deg}(C)=(\text { number of cups in } C) \pm 1 \text {, }
$$

with +1 , if the circle $C$ is oriented clockwise and -1 otherwise. 
(b) If, in an oriented circle diagram $\underline{\lambda} \nu \bar{\mu}$, one changes the orientation such that all vertices contained in exactly one circle $C$ are changed, then the degree increases by 2 , if $C$ was oriented anticlockwise, and decreases by 2 , if $C$ was oriented clockwise.

Proof. (a) is a special case of [12, Proposition 4.9] while (b) follows from (a).

We conclude this part with the notions of distance and saddle width, which will be important for spreading signs in the multiplication given below.

Definition 3.10. For $i \in \mathbb{Z}$ and a block $\Lambda$ define the position of $i$ as

$$
\mathrm{p}_{\Lambda}(i)=\left|\left\{j \mid j \leq i, \operatorname{seq}(\Lambda)_{j}=\star\right\}\right|+2\left|\left\{j \mid j \leq i, \operatorname{seq}(\Lambda)_{j}=\times\right\}\right| .
$$

For a cup or cap $\gamma$ in a diagram connecting vertices $(i, 0)$ and $(j, 0)$ we define its distance $\mathrm{d}_{\Lambda}(\gamma)$ and saddle width $s_{\Lambda}(\gamma)$ by

$$
\mathrm{d}_{\Lambda}(\gamma)=\left|\mathrm{p}_{\Lambda}(i)-\mathrm{p}_{\Lambda}(j)\right| \quad \text { respectively } \quad s_{\Lambda}(\gamma)=\frac{1}{2}\left(\mathrm{~d}_{\Lambda}(\gamma)+1\right)
$$

For a ray $\gamma$ set $\mathrm{d}_{\Lambda}(\gamma)=0$. For a collection $M=\left\{\gamma_{1}, \ldots, \gamma_{r}\right\}$ of distinct arcs (e.g. a circle or sequence of arcs connecting two vertices) set

$$
\mathrm{d}_{\Lambda}(M)=\sum_{1 \leq k \leq r} \mathrm{~d}_{\Lambda}\left(\gamma_{k}\right)
$$

The saddle width will be interpreted in Subsection 4.4 as the number of phantom facets at the bottom of a saddle (e.g. $s=2$ for the saddle from (21)).

We omit the subscript $\Lambda$, if no confusion can arise.

3.2. The Blanchet-Khovanov algebras as graded $\mathbb{K}$-vector spaces. Fix a block $\Lambda \in \mathrm{bl}$, and consider the basis set of oriented circle diagrams

$$
\mathbb{B}(\Lambda)=\{\underline{\lambda} \nu \bar{\mu} \mid \underline{\lambda} \nu \bar{\mu} \text { is oriented and } \lambda, \mu, \nu \in \Lambda\} .
$$

This set is subdivided into smaller sets of the form ${ }_{\lambda} \mathbb{B}(\Lambda)_{\mu}$ which are those diagrams in $\mathbb{B}(\Lambda)$ which have $\underline{\lambda}$ as cup part and $\bar{\mu}$ as cap part.

From now on, we restrict to circle diagrams that only contain cups and caps. Formally this is done as follows: for a block $\Lambda \in \mathrm{bl}$ denote by $\Lambda^{\circ}$ the set of weights $\lambda$ such that $\underline{\lambda}$ only contains cups. Note that $\Lambda^{\circ} \neq \emptyset$ iff $\Lambda$ is balanced. Define

$$
\mathbb{B}^{\circ}(\Lambda)=\left\{\underline{\lambda} \nu \bar{\mu} \mid \underline{\lambda} \nu \bar{\mu} \text { is oriented and } \lambda, \mu \in \Lambda^{\circ}, \nu \in \Lambda\right\}=\bigcup_{\lambda, \mu \in \Lambda^{\circ}}{ }_{\lambda} \mathbb{B}(\Lambda)_{\mu} .
$$

Definition 3.11. The Blanchet-Khovanov algebra $\mathfrak{A}_{\Lambda}^{\mathfrak{F}}$ attached to a block $\Lambda \in \mathrm{bl}{ }^{\diamond}$ and the (full) Blanchet-Khovanov algebra $\mathfrak{A}^{\mathfrak{F}}$ are the graded $\mathbb{K}$-vector space

$$
\mathfrak{A}_{\Lambda}^{\mathfrak{F}}=\left\langle\mathbb{B}^{\circ}(\Lambda)\right\rangle_{\mathbb{K}}=\bigoplus_{(\underline{\lambda} \nu \bar{\mu}) \in \mathbb{B}^{\circ}(\Lambda)} \mathbb{K}(\underline{\lambda} \nu \bar{\mu}), \quad \mathfrak{A}^{\mathfrak{F}}=\bigoplus_{\Lambda \in \mathrm{b} 1^{\diamond}} \mathfrak{A}_{\Lambda}^{\mathfrak{F}},
$$

with multiplication mult given in Subsection 3.3 . Denote also by ${ }_{\lambda}\left(\mathfrak{A}_{\Lambda}^{\mathfrak{F}}\right)_{\mu}$ the span of the basis vectors inside ${ }_{\lambda} \mathbb{B}(\Lambda)_{\mu}$.

Proposition 3.12. The map mult: $\mathfrak{A}_{\Lambda}^{\mathfrak{F}} \otimes \mathfrak{A}_{\Lambda}^{\mathfrak{F}} \rightarrow \mathfrak{A}_{\Lambda}^{\mathfrak{F}}$ given in Subsection 3.3 endows $\mathfrak{A}_{\Lambda}^{\mathfrak{F}}$ with the structure of a graded, unital algebra with pairwise orthogonal, primitive idempotents ${ }_{\lambda} \mathbb{1}_{\lambda}=\underline{\lambda} \lambda \bar{\lambda}$ for $\lambda \in \Lambda$ and unit $\mathbb{1}=\sum_{\lambda \in \Lambda} \mathbb{1}_{\lambda}$. Similar for (the locally unital) algebra $\mathfrak{A}^{\mathfrak{F}}$.

Proof. The maps mult ${ }_{D_{l}, D_{l+1}}$ are homogeneous of degree 0 by [12, Proposition 5.19], since the proof is diagrammatic and independent of any signs or coefficients. The proof that the ${ }_{\lambda} \mathbb{1}_{\lambda}$ are idempotents is the same as in [12, Theorem 6.2], since multiplying them only involves merges of non-nested circles, in which case the map mult $_{D_{l}, D_{l+1}}$ agrees with the one defined in [12, Section 5]. That they are pairwise orthogonal and primitive is clear by definition. 
Remark 3.13. Note that so far we do not know whether $\mathfrak{A}_{\Lambda}^{\mathfrak{F}}$ is associative. It will follow from the identification of $\mathfrak{A}_{\Lambda}^{\mathfrak{F}}$ with $\mathfrak{W}_{\vec{k}}$ that mult is independent of the chosen order in which the surgeries are performed and that $\mathfrak{A}_{\Lambda}^{\mathfrak{F}}$ is associative, see Corollary 4.19. Alternatively, the independence from the chosen order and associativity can be shown in the same spirit as [12, Theorem 5.34].

3.3. Multiplication of the Blanchet-Khovanov algebra. The multiplication on $\mathfrak{A}_{\Lambda}^{\mathfrak{F}}$ is based on the one defined in [18] and used in [4]. We will first recall the maps used in each step, which are the same as in 4] and afterwards go into details about how we modify these maps with different sign choices.

For $\lambda, \mu, \mu^{\prime}, \eta \in \Lambda^{\circ}$ we define a map mult : ${ }_{\lambda}\left(\mathfrak{A}_{\Lambda}^{\mathfrak{F}}\right)_{\mu} \otimes_{\mu^{\prime}}\left(\mathfrak{A}_{\Lambda}^{\mathfrak{F}}\right)_{\eta} \rightarrow{ }_{\lambda}\left(\mathfrak{A}_{\Lambda}^{\mathfrak{F}}\right)_{\eta}$ as follows. If $\mu \neq \mu^{\prime}$ we declare the map to be identically zero. Thus, assume that $\mu=\mu^{\prime}$, and stack the diagram, without orientations, $\mu \bar{\eta}$ on top of the diagram $\underline{\lambda} \bar{\mu}$, creating a diagram $D_{0}=\underline{\lambda} \bar{\mu} \mu \bar{\eta}$. In [12, Definition 5.1] such a diagram is called a stacked circle diagram. Given such a diagram $D_{l}$, starting with $l=0$, we construct below a new diagram $D_{l+1}$ by choosing a certain symmetric pair of a cup and a cap in the middle section. If $r$ is the number of cups in $\mu$, then this can be done a total number of $r$ times. We call this procedure a surgery at the corresponding cup-cap pair. For each such step we define below a map mult D $_{l}, D_{l+1}$. Observing that the space of orientations of the final diagram $D_{r}$ is equal to the space of orientations of the diagram $\underline{\lambda} \bar{\eta}$, we define

$$
\text { mult }=\text { mult }_{D_{r-1}, D_{r}} \circ \ldots \circ \text { mult }_{D_{0}, D_{1}}:{ }_{\lambda}\left(\mathfrak{A}_{\Lambda}^{\mathfrak{F}}\right)_{\mu} \otimes \mu_{\mu^{\prime}}\left(\mathfrak{A}_{\Lambda}^{\mathfrak{F}}\right)_{\eta} \rightarrow_{\lambda}\left(\mathfrak{A}_{\Lambda}^{\mathfrak{F}}\right)_{\eta} .
$$

The global map mult is defined as the direct sum of all the ones defined here. In order to make mult a priori well-defined, we always pick the leftmost available cup-cap pair. Corollary 4.19 will finally ensure that this fixed choice is irrelevant.

3.3.1. The surgery procedure. To obtain $D_{l+1}$ from $D_{l}=\underline{\lambda} c^{*} c \bar{\eta}$ (for some cup diagram $c$ ) choose the cup-cap pair with the leftmost endpoint in $c^{*} c$ that can be connected without crossing any arcs (this means that the cup and cap are not nested inside any other arcs). Cut open the cup and the cap and stitch the loose ends together to form a pair of vertical line segments, call this diagram $D_{l+1}$ :

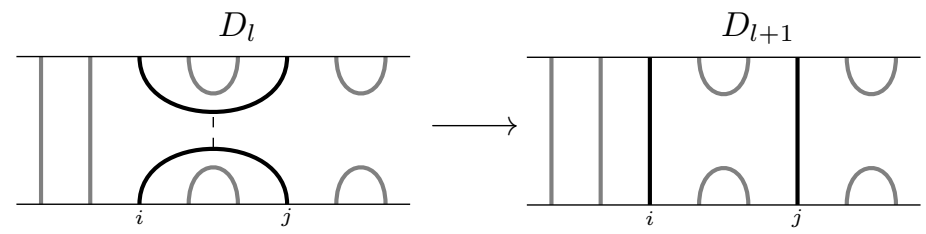

3.3.2. The map without signs. As in [18] and [4] the map mult (D) $_{D_{l+1}, D_{l}}$, without any additional signs only depends on how the components change when going from $D_{l}$ to $D_{l+1}$. The image of an orientation of $D_{l}$ is constructed as follows in these cases (where we always leave the orientations on non-interacting arcs fixed).

Merge. If two circles, say $C_{1}$ and $C_{2}$, are merged into a circle $C$ proceed as follows. - If both circles are oriented anticlockwise, then orient $C$ anticlockwise.

- If one circle is oriented clockwise, one is oriented anticlockwise, then orient $C$ clockwise.

- If both circles are oriented clockwise, then the map is zero.

Split. If one circle $C$ is replaced by two circles, say $C_{1}$ and $C_{2}$, proceed as follows.

- If $C$ is oriented anticlockwise, then take two copies of $D_{l+1}$. In one copy orient

$C_{1}$ clockwise and $C_{2}$ anticlockwise, in the other vice versa.

- If $C$ is oriented clockwise, then orient both, $C_{1}$ and $C_{2}$, clockwise. 
Remark 3.14. These are the same rules as in [18] and 44. They can also be given by a TQFT, but the direct definition simplifies the introduction of signs.

3.3.3. The map with signs. In general, the formulas below include two types of signs. One type, which we call dot moving signs, also appear in [12, while the second, called topological signs and saddle signs, are topological in nature and more involved. These second types of signs will be given an interesting meaning in Subsection 4.5. The main difference to 12 will be that we distinguish whether the two circles, that are merged together or split into, are nested in each other or not. Define:

$$
\mathrm{t}(C)=\text { (a choice of) a rightmost point in the circle } C \text {. }
$$

Let $\gamma$ denote the cup in the cup-cap pair we use to perform the surgery procedure in this step connecting vertices $i<j$.

Non-nested Merge. The non-nested circles $C_{1}$ and $C_{2}$ are merged into $C$. The only case that is modified here is:

- One circle oriented clockwise, one oriented anticlockwise. Let $C_{k}$ (for $k \in\{1,2\}$ ) be the clockwise oriented circle and let $\gamma^{\text {dot }}$ be a sequence of arcs in $C$ connecting $\mathrm{t}\left(C_{k}\right)$ and $\mathrm{t}(C)$. (Neither $\mathrm{t}\left(C_{k}\right), \mathrm{t}(C)$ nor $\gamma^{\text {dot }}$ are unique, but possible choices differ in distance by 2 , making the sign well-defined, see also [12, Lemma 5.7]. Thus, the reader may choose any of these.) Proceed as in Subsection 3.3.2 and multiply with the dot moving sign

$$
(-1)^{\mathrm{d}_{\Lambda}\left(\gamma^{\mathrm{dot}}\right)}
$$

Nested Merge. The nested circles $C_{1}$ and $C_{2}$ are merged into $C$. Denote by $C_{\text {in }}$ the inner of the two original circles. The cases are modified as follows.

- Both circles oriented anticlockwise. Proceed as above, but multiply with

$$
-(-1)^{\frac{1}{4}\left(\mathrm{~d}_{\Lambda}\left(C_{\mathrm{in}}\right)-2\right)} \cdot(-1)^{s_{\Lambda}(\gamma)}
$$

- One circle oriented clockwise, one oriented anticlockwise. Again perform the surgery procedure as described in Subsection 3.3.2 and multiply with

$$
(-1)^{\mathrm{d}_{\Lambda}\left(\gamma^{\mathrm{dot}}\right)} \cdot\left(-(-1)^{\frac{1}{4}\left(\mathrm{~d}_{\Lambda}\left(C_{\mathrm{in}}\right)-2\right)}\right) \cdot(-1)^{s_{\Lambda}(\gamma)},
$$

where $\gamma^{\text {dot }}$ is defined as in 25 .

Non-nested Split. The circle $C$ splits into the non-nested circles $C_{i}$, containing the vertices at position $i$, and $C_{j}$, containing the vertices at position $j$. For both orientations we introduce a dot moving sign as well as a saddle sign as follows.

- $C$ oriented anticlockwise. Use the map as in Subsection 3.3.2 but the copy where $C_{i}$ is oriented clockwise is multiplied with

$$
(-1)^{\mathrm{d}_{\Lambda}\left(\gamma_{i}^{\text {ndot }}\right)} \cdot(-1)^{s_{\Lambda}(\gamma)},
$$

while the one where $C_{j}$ is oriented clockwise is multiplied with

$$
-(-1)^{\mathrm{d}_{\Lambda}\left(\gamma_{j}^{\text {ndot }}\right)} \cdot(-1)^{s_{\Lambda}(\gamma)} .
$$

Here $\gamma_{i}^{\text {ndot }}$ and $\gamma_{j}^{\text {ndot }}$ are sequences of arcs connecting $(i, 0)$ and $\mathrm{t}\left(C_{i}\right)$ inside $C_{i}$ respectively $(j, 0)$ and $\mathrm{t}\left(C_{j}\right)$ in $C_{j}$ (ndot can be read as "newly created dot").

- $C$ oriented clockwise. In this case multiply the result with

$$
(-1)^{\mathrm{d}_{\Lambda}\left(\gamma^{\mathrm{dot}}\right)} \cdot(-1)^{\mathrm{d}_{\Lambda}\left(\gamma_{i}^{\text {ndot }}\right)} \cdot(-1)^{s_{\Lambda}(\gamma)} \cdot
$$

Here $\gamma^{\text {dot }}$ is a sequence of arcs connecting $\mathrm{t}(C)$ and $\mathrm{t}\left(C_{j}\right)$ in $C$ and $\gamma_{i}^{\text {ndot }}$ is as before. 
Nested Split. We use here the same notations as in the non-nested split case above and furthermore denote by $C_{\text {in }}$ the inner of the two circles $C_{i}$ and $C_{j}$. The difference to the non-nested case is that we substitute the saddle sign with a topological sign. - $C$ oriented anticlockwise. We use the map as defined in Subsection 3.3.2, but the copy where $C_{i}$ is oriented clockwise is multiplied with

$$
(-1)^{\mathrm{d}_{\Lambda}\left(\gamma_{i}^{\text {ndot }}\right)} \cdot(-1)^{\frac{1}{4}\left(\mathrm{~d}_{\Lambda}\left(C_{\mathrm{in}}\right)-2\right)}
$$

while the copy where $C_{j}$ is oriented clockwise is multiplied with

$$
-(-1)^{\mathrm{d}_{\Lambda}\left(\gamma_{j}^{\text {ndot }}\right)} \cdot(-1)^{\frac{1}{4}\left(\mathrm{~d}_{\Lambda}\left(C_{\mathrm{in}}\right)-2\right)} .
$$

Here $\gamma_{i}^{\text {ndot }}$ and $\gamma_{j}^{\text {ndot }}$ are as before.

- $C$ oriented clockwise. We multiply with

$$
(-1)^{\mathrm{d}_{\Lambda}\left(\gamma^{\mathrm{dot}}\right)} \cdot(-1)^{\mathrm{d}_{\Lambda}\left(\gamma_{i}^{\mathrm{ndot}}\right)} \cdot(-1)^{\frac{1}{4}\left(\mathrm{~d}_{\Lambda}\left(C_{\text {in }}\right)-2\right)},
$$

again with the same notations as above.

3.3.4. Examples for the multiplication. We give below examples for some of the shapes that can occur during the surgery procedure and determine the signs. In all examples assume that outside of the shown strip all entries of the weights are $\circ$.

Example 3.15. This example illustrates the merge situation. First we look at a simple merge of two anticlockwise, non-nested circles. In this case no signs appear at all that means we have

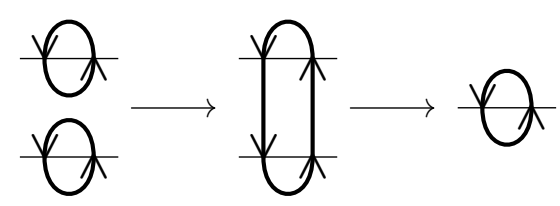

The rightmost step above, called collapsing, is always performed at the end of a multiplication procedure and is omitted in what follows.

Secondly, we consider a merge of two anticlockwise, nested circles. Depending on the concrete shape of the diagram it can produce different signs:

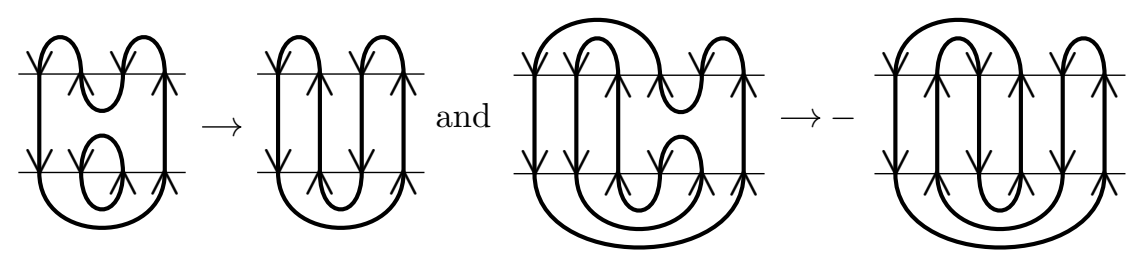

Note that, in contrast to [18, 4] or [12, nested merges can come with a sign.

Example 3.16. This example illustrated the two versions of a split. In both cases a non-nested merge is performed, followed by a split into two non-nested respectively nested circles. First, the H-shape: 
$(29)$
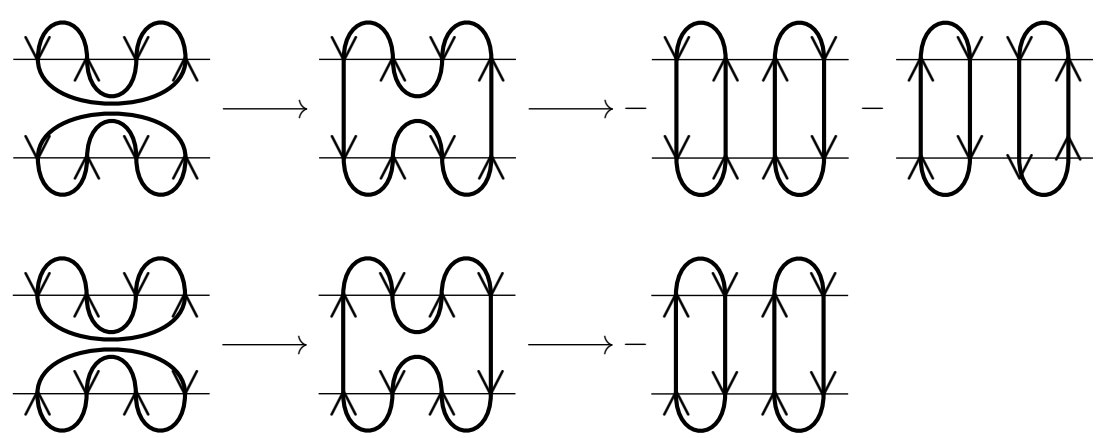

Next, the C shape:
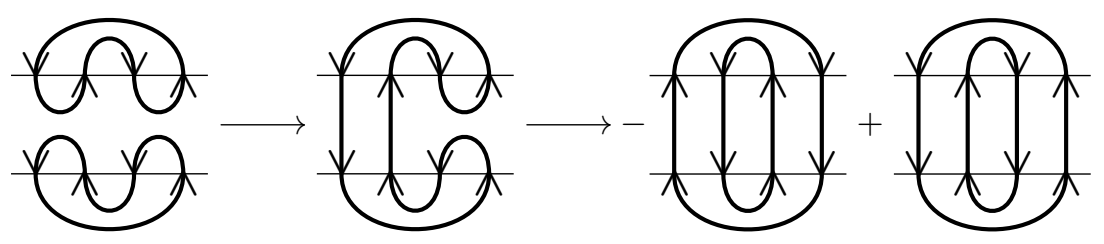

$(30)$

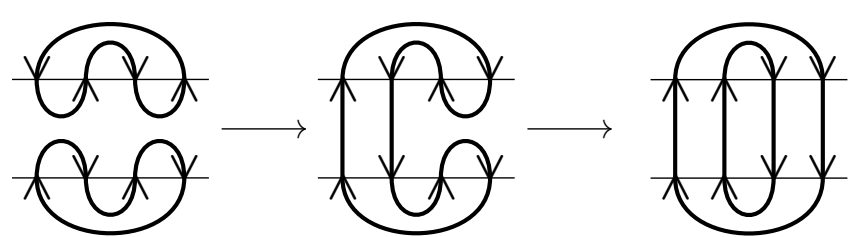

Remark 3.17. The $\supset$ shape cannot appear as long as we impose the choice of the order of cup-cap pairs from left to right in the surgery procedure and it will not be needed in the proof of Theorem 4.18 .

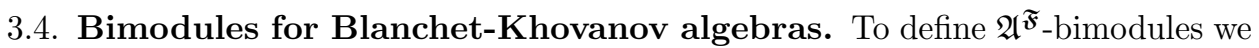
need further diagrams moving from one block $\Lambda$ to another block $\Gamma$.

Fix two blocks $\Lambda, \Gamma \in \mathrm{bl}^{\diamond}$ such that $\operatorname{seq}(\Lambda)$ and $\operatorname{seq}(\Gamma)$ coincide except at positions $i$ and $i+1$. Following [5], a $(\Lambda, \Gamma)$-admissible matching (of type $\pm \alpha_{i}$ ) is a diagram $t$ inside $\mathbb{R} \times[0,1]$ consisting of vertical lines connecting $(k, 0)$ with $(k, 1)$ if we have that $\operatorname{seq}(\Lambda)_{k}=\operatorname{seq}(\Gamma)_{k}=\star$ and, depending on the sign of $\alpha_{i}$, an arc at positions $i$ and $i+1$ of the form
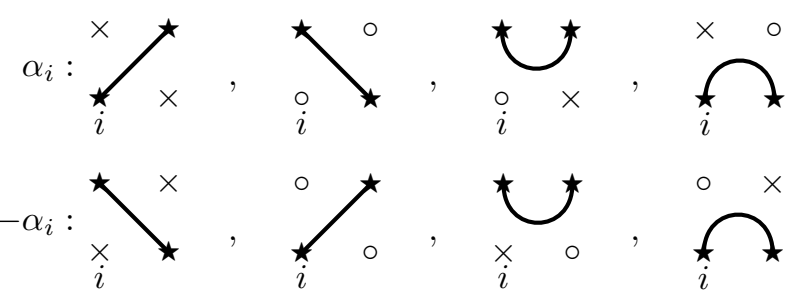

where we view seq $(\Lambda)$ as decorating the integral points of $\mathbb{R} \times\{0\}$ and seq $(\Gamma)$ as decorating the integral points of $\mathbb{R} \times\{1\}$. Again, the first two moves in each row are called rays, the third ones cups and the last ones caps.

For $t$ a $(\Lambda, \Gamma)$-admissible matching, $\lambda \in \Lambda$, and $\mu \in \Gamma$ we say that $\lambda t \mu$ is oriented if cups respectively caps connect one $\wedge$ and one $\vee$ in $\lambda$ respectively $\mu$, and rays connect the same symbols in $\lambda$ and $\mu$. For $\boldsymbol{\Lambda}=\left(\Lambda_{0}, \ldots, \Lambda_{r}\right)$ a sequence of blocks 
a $\boldsymbol{\Lambda}$-admissible composite matching is a sequence of diagrams $\mathbf{t}=\left(t_{1}, \ldots, t_{r}\right)$ such that $t_{k}$ is a $\left(\Lambda_{k-1}, \Lambda_{k}\right)$-admissible matching of some type. We view the sequence of matchings as being stacked on top of each other. A sequence of weights $\lambda_{i} \in \Lambda_{i}$ such that $\lambda_{k-1} t_{k} \lambda_{k}$ is oriented for all $k$ is an orientation of the $\boldsymbol{\Lambda}$-admissible composite matching $\mathbf{t}$. For short, we tend to drop the word admissible, since the only matchings we consider are admissible. Moreover, if only start $\Lambda=\Lambda_{0}$ and end $\Gamma=\Lambda_{r}$ matter, then we call $\mathbf{t}$ short a $(\Lambda, \Gamma)$-composite matching.

We stress that $\boldsymbol{\Lambda}$-composite matching can contain lines, in contrast to the diagrams we consider for $\mathfrak{A}_{\Lambda}^{\mathfrak{F}}$ and $\mathfrak{A}^{\mathfrak{F}}$.

Example 3.18. Below is a $\left(\Lambda_{0}, \Lambda_{5}\right)$-composite matching. Assume that outside of the indicated areas all symbols of the block sequences are equal to $\circ$.

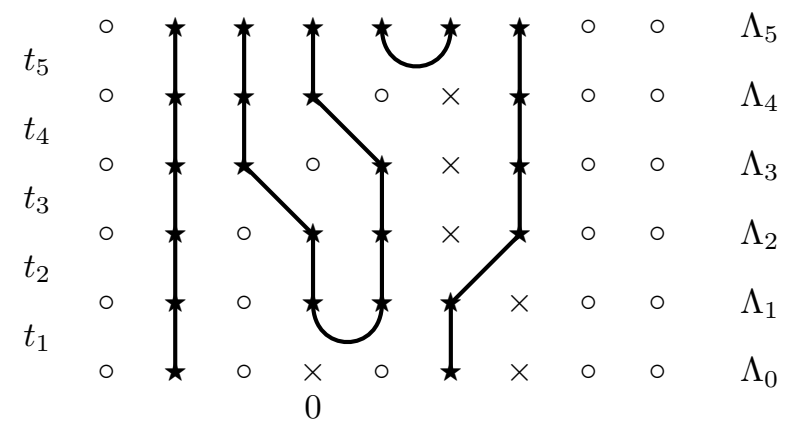

The types of the matchings are $-\alpha_{0}, \alpha_{2}, \alpha_{-1}, \alpha_{0}, \alpha_{1}$ (read from bottom to top).

We now want to consider bimodules between Blanchet-Khovanov algebras for different blocks, or said differently, bimodules for the algebra $\mathfrak{A}^{\mathfrak{F}}$.

We start by defining a basis of the underlying $\mathbb{K}$-vector space. To a $\boldsymbol{\Lambda}$-composite matching $\mathbf{t}$ we again associate a set of diagrams from which to create a $\mathbb{K}$-vector space (its elements are called stretched circle diagrams)

$$
\mathbb{B}^{\circ}(\boldsymbol{\Lambda}, \mathbf{t})=\left\{\begin{array}{l|l}
\underline{\lambda}(\mathbf{t}, \boldsymbol{\nu}) \bar{\mu} & \begin{array}{l}
\lambda \in \Lambda_{0}^{\circ}, \mu \in \Lambda_{r}^{\circ}, \boldsymbol{\nu}=\left(\nu_{0}, \ldots, \nu_{r}\right) \text { with } \nu_{i} \in \Lambda_{i}, \\
\underline{\lambda} \nu_{0} \text { oriented, } \nu_{r} \bar{\mu} \text { oriented, } \\
\nu_{i-1} t_{i} \nu_{i} \text { oriented for all } 1 \leq i \leq r .
\end{array}
\end{array}\right\}
$$

As before we obtain the set $\mathbb{B}(\boldsymbol{\Lambda}, \mathbf{t})$ by allowing $\lambda \in \Lambda_{0}$ and $\mu \in \Lambda_{r}$ in $(32)$.

Example 3.19. Let $\Lambda$ be the block with block sequence $\star \star \circ \times$ at positions $0,1,2,3$ and $\Gamma$ the block with sequence $\star \star \star \star \star$ at the same positions (both with $\circ$ everywhere else) and assume both blocks are balanced. Then an example for a $(\Lambda, \Gamma)$-matching of type $\alpha_{1}$ is the third diagram in the first row of (31) denoted here by $t_{1}$. Taking this as our composite matching we obtain a $\mathbb{K}$-vector space of dimension 6 with basis consisting of
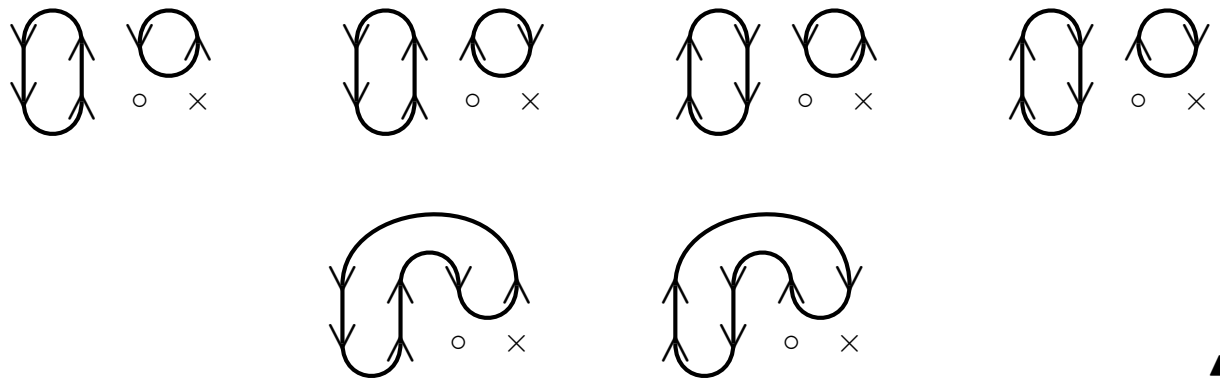

For a basis vector $\mu(\mathbf{t}, \boldsymbol{\nu}) \bar{\eta}$ denote by $\bar{\eta}^{\downarrow}$ its downwards reduction. This is the cap diagram obtained by stacking the diagrams $t_{1}, \ldots, t_{r}, \bar{\eta}$ on top of each other from left 
to right, removing any components in this stacked diagram that are not connected to the bottom line of $t_{1}$, and replacing all components that are connected to the bottom in two vertices by a cap connecting the vertices. It is clearly independent of $\underline{\mu}$. Analogously, define its upward reduction $\underline{\mu}^{\uparrow}$, a cup diagram independent of $\bar{\eta}$.

Definition 3.20. Let $\mathbf{t}$ be a $\boldsymbol{\Lambda}$-composite matching for $\boldsymbol{\Lambda}=\left(\Lambda_{0}, \ldots, \Lambda_{r}\right)$. Set

$$
\mathfrak{A}^{\mathfrak{F}}(\boldsymbol{\Lambda}, \mathbf{t})=\left\langle\mathbb{B}^{\circ}(\boldsymbol{\Lambda}, \mathbf{t})\right\rangle_{\mathbb{K}}
$$

as a graded $\mathbb{K}$-vector space. Hereby the degree of a basis element itself is, by definition, minus the number of its anticlockwise circles plus the number of its clockwise circles.

For a basis element $\underline{\lambda} \nu \bar{\mu} \in \mathfrak{A}_{\Lambda_{0}}^{\mathfrak{F}}$ define a left (bottom) action via

$$
(\underline{\lambda} \nu \bar{\mu})(\underline{\mu}(\mathbf{t}, \boldsymbol{\nu}) \bar{\eta})=\sum_{\boldsymbol{\nu}^{\prime}} a_{\boldsymbol{\nu}^{\prime}} \underline{\lambda}\left(\mathbf{t}, \boldsymbol{\nu}^{\prime}\right) \bar{\eta},
$$

with the coefficients $a_{\nu^{\prime}}$ given by the multiplication

$$
(\underline{\lambda} \nu \bar{\mu})\left(\underline{\mu} \nu_{0} \bar{\eta}^{\downarrow}\right)=\sum_{\nu^{\prime}} a_{\nu^{\prime}} \underline{\lambda} \nu^{\prime} \bar{\eta}^{\downarrow}
$$

Then $a_{\nu^{\prime}}=a_{\nu^{\prime}}$ for $\nu^{\prime}$ the unique orientation with $\nu_{0}^{\prime}=\nu^{\prime}$ and all components not connected to the bottom line of $t_{1}$ have the same orientation as in $\mu(\mathbf{t}, \boldsymbol{\nu}) \bar{\eta}$. Analogously define a right (top) action of $\mathfrak{A}_{\Lambda_{r}}^{\mathfrak{F}}$ using the upwards reduction $\underline{\mu}^{\uparrow}$.

Example 3.21. The basis elements (read from left to right) from Example 3.19 have degrees $-2,0,0,2$ (top row) and $-1,1$ (bottom row).

It is not clear that the above actions are well-defined and commute and we need the translation between $\mathfrak{W}$ and $\mathfrak{A}^{\mathfrak{F}}$ from Section 4 to prove it.

Proposition 3.22. Let $\mathbf{t}$ be a $\boldsymbol{\Lambda}$-composite matching for $\boldsymbol{\Lambda}=\left(\Lambda_{0}, \ldots, \Lambda_{r}\right)$. Then the left action of $\mathfrak{A}_{\Lambda_{0}}^{\mathfrak{F}}$ and the right action of $\mathfrak{A}_{\Lambda_{r}}^{\mathfrak{F}}$ on $\mathfrak{A}^{\mathfrak{F}}(\boldsymbol{\Lambda}, \mathbf{t})$ are well-defined and commute. Hence, $\mathfrak{A}^{\mathfrak{F}}(\boldsymbol{\Lambda}, \mathbf{t})$ is a $\mathfrak{A}_{\Lambda_{0}}^{\mathfrak{F}}-\mathfrak{A}_{\Lambda_{r}}^{\mathfrak{F}}$-bimodule (and thus, a $\mathfrak{A}^{\mathfrak{F}}$-bimodule).

Proof. Using Theorem 4.18, we obtain an isomorphism of graded algebras of $\mathfrak{A}_{\Lambda}^{\mathfrak{F}}$ with $\mathfrak{W}_{\vec{k}}^{\circ}$. An isomorphism of graded $\mathbb{K}$-vector spaces of $\mathfrak{A}^{\mathfrak{F}}(\boldsymbol{\Lambda}, \mathbf{t})$ with $\mathfrak{W}(\mathbf{w}(\boldsymbol{\Lambda}, \mathbf{t}))$ is obtained by using Lemma 4.16. This isomorphism intertwines the actions of the two algebras on the bimodules by construction and hence, proves the claim.

We introduce a slight generalization of the notion of an admissible matching, the so-called empty moves (of which the reader should think of switching neighboring o and $\times$ ). This means that in the list of local moves $(31$ we also allow the following:
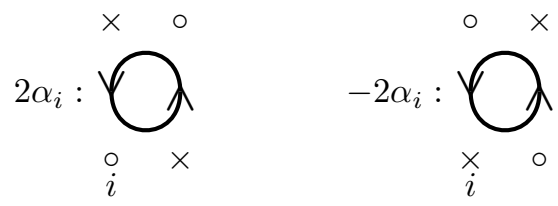

If a composite matching $\mathbf{t}$ contains empty moves, $\mathfrak{A}^{\mathfrak{F}}(\boldsymbol{\Lambda}, \mathbf{t})$ is constructed as follows. Take the composite matching $\mathbf{t}^{\prime}$ that is obtained by substituting each empty move by the composition of a cup and cap local move such that its fits with the two blocks. Then take the submodule spanned by those basis elements such that the internal circles are all oriented anticlockwise. It is evident that this will be a submodule of the full bimodule where all orientations are allowed. Finally, shift the module up by the total number of empty moves in $\mathbf{t}$. 
Example 3.23. The pictures below are wildcards for the $\mathfrak{A}^{\mathfrak{F}}$-bimodules defined via the illustrated matchings.

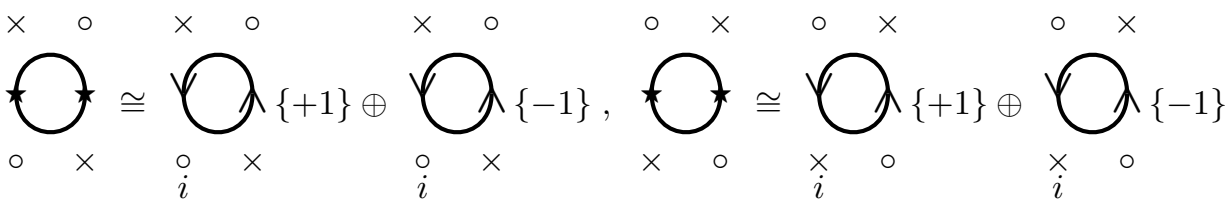

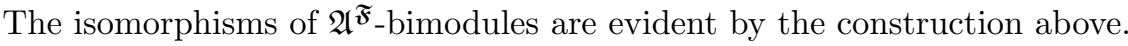

Proposition 3.24. The $\mathfrak{A}^{\mathfrak{F}_{\text {-bimodules }}} \mathfrak{A}^{\mathfrak{F}}(\boldsymbol{\Lambda}, \mathbf{t})$ are finite-dimensional, graded biprojective $\mathfrak{A}^{\mathfrak{F}}$-bimodules.

Proof. Clearly, $\mathfrak{A}^{\mathfrak{F}}(\boldsymbol{\Lambda}, \mathbf{t})$ are finite-dimensional $\mathfrak{A}^{\mathfrak{F}}$-bimodules. That they are graded as $\mathfrak{A}^{\mathfrak{F}}$-bimodules follows by the identification with web bimodules from Lemma 4.16 We only prove here projectivity for the left action, the right action is done analogously. Denote by $\Lambda$ and $\Gamma$ the first and last block in $\Lambda$. For any $\mu \in \Gamma$ denote by $\mu_{\mu \downarrow} \mathbb{1}_{\mu \downarrow}$ the idempotent in $\mathfrak{A}_{\Lambda}^{\mathfrak{F}}$ corresponding to the downwards reduction of $\mu$. Then, as an $\mathfrak{A}_{\Lambda}^{\mathfrak{F}}$-module, $\mathfrak{A}^{\mathfrak{F}}(\boldsymbol{\Lambda}, \mathbf{t})$ decomposes as a direct sum of modules of the form $\mathfrak{A}_{\Lambda}^{\mathfrak{F}} \cdot\left({ }_{\mu \downarrow} \mathbb{1}_{\mu^{\downarrow}}\right)$, which are projective $\mathfrak{A}_{\Lambda}^{\mathfrak{F}}$-modules, proving the claim.

This proposition again motivates the definition of the following 2-category which is, as before, one of the main objects that we are going to study.

Definition 3.25. Given $\mathfrak{A}^{\mathfrak{F}}$ as above, let $\mathfrak{A}^{\mathfrak{F}}$-biMod ${ }_{\text {gr }}^{p}$ be the following 2-category:

- Objects are the various $\Lambda \in \mathrm{bl}^{\diamond}$.

- Morphisms are finite sums and tensor products (taken over $\mathfrak{A}^{\mathfrak{F}}$ ) of the $\mathfrak{A}^{\mathfrak{F}}$-bimodules $\mathfrak{A}^{\mathfrak{F}}(\boldsymbol{\Lambda}, \mathbf{t})$.

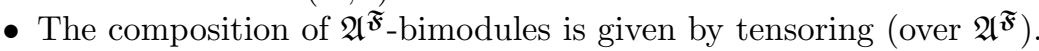

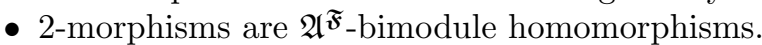

- The vertical composition of $\mathfrak{A}^{\mathfrak{F}}$-bimodule homomorphisms is the usual composition and the horizontal composition is given by tensoring (over $\mathfrak{A}^{\mathfrak{F}}$ ).

We consider $\mathfrak{A}^{\mathfrak{F}_{\text {-biMod }}} \mathbf{g r}_{\text {gr }}^{p}$ as a graded 2-category with 2-hom-spaces as in (1).

In analogy to Web from the end of Subsection 2.2, the objects and morphisms (when seen as composite matchings) in $\mathfrak{A}^{\mathfrak{F}_{\text {-biMod }}} \mathbf{d}_{\text {gr }}^{p}$ can be seen as a $\mathbb{K}(q)$-linear category, which we denote by $\mathbf{C M}$.

\section{Equivalences}

In this section we assume that all appearing $\vec{k}$ 's and $\Lambda$ 's are balanced. Our goal now is to construct an isomorphism of graded algebras $\Phi: \mathfrak{W}^{\circ} \rightarrow \mathfrak{A}^{\mathfrak{F}}$ (where $\mathfrak{W}^{\circ}$ is a certain subalgebra of $\mathfrak{W}$ defined in 40 ). This isomorphism $\Phi$ induces the following:

Theorem 4.1. There is an equivalence of graded, $\mathbb{K}$-linear 2-categories

$$
\boldsymbol{\Phi}: \mathfrak{W}-\text { biMod }_{\mathrm{gr}}^{p} \stackrel{\cong}{\longrightarrow} \mathfrak{A}^{\mathfrak{F}_{-}} \text {biMod }_{\mathrm{gr}}^{p}
$$

under which the $\mathfrak{W}$-bimodules $\mathfrak{W}(\mathbf{w}(\boldsymbol{\Lambda}, \mathbf{t})$ ) (with $\mathbf{w}(\boldsymbol{\Lambda}, \mathbf{t})$ defined in Definition 4.9 below) are identified with the $\mathfrak{A}^{\mathfrak{F}_{\text {-bimodules }}} \mathfrak{A}^{\mathfrak{F}}(\boldsymbol{\Lambda}, \mathbf{t})$.

4.1. Some useful lemmas. In order to prove Theorem 4.1, we need some lemmas.

Lemma 4.2. Let $\ell \in \mathbb{Z}_{\geq 0}$. Then $\operatorname{dim}_{\mathbb{K}}\left(2 \operatorname{End}_{\mathfrak{F}}\left(\mathbf{1}_{2 \omega_{\ell}}\right)\right)=1$.

Proof. Note that the identity foam on $\mathbf{1}_{2 \omega_{\ell}}$ (i.e. $\ell$ parallel phantom facets) is an element of $2 \operatorname{End}_{\mathfrak{F}}\left(\mathbf{1}_{2 \omega_{\ell}}\right)$ which shows that $\operatorname{dim}_{\mathbb{K}}\left(2 \operatorname{End}_{\mathfrak{F}}\left(\mathbf{1}_{2 \omega_{\ell}}\right)\right) \geq 1$. Now, given any $f \in 2 \operatorname{End}_{\mathfrak{F}}\left(\mathbf{1}_{2 \omega_{\ell}}\right)$, denote by $g \in 2 \operatorname{End}_{\overline{\mathfrak{F}}}(\emptyset)$ (where $\emptyset$ denotes the empty web) the closed foam obtained from $f$ by closings the $\ell$ bottom and top phantom facets to 
phantom spheres. Note that the question whether there are relations to reduce $f$ to a scalar multiple of the identity foam on $\mathbf{1}_{2 \omega_{\ell}}$ is the same as the question whether $g$ can be evaluated to a scalar. But the latter can be achieved using the relations in $\overline{\mathfrak{F}}$ (which can be shown similar as in [19, Proposition 5]). Thus, every such $f$ is a scalar multiple of the identity foam on $\mathbf{1}_{2 \omega_{\ell}}$ which shows that $\operatorname{dim}_{\mathbb{K}}\left(2 \operatorname{End}{ }_{\mathfrak{F}}\left(\mathbf{1}_{2 \omega_{\ell}}\right)\right) \leq 1$.

Lemma 4.3. The following foams are locally one-sided invertible in $\mathfrak{F}$ (the ones in the first column have a right inverse obtained by gluing from the bottom, the ones in the second column a left inverse obtained by gluing from the top):
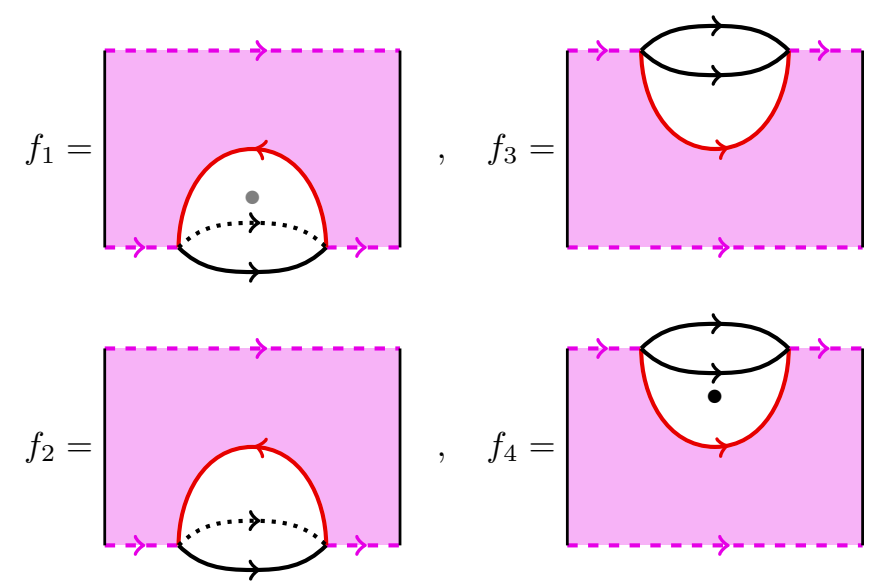

Similarly with the dots moved to the opposite facets. This implies locally that

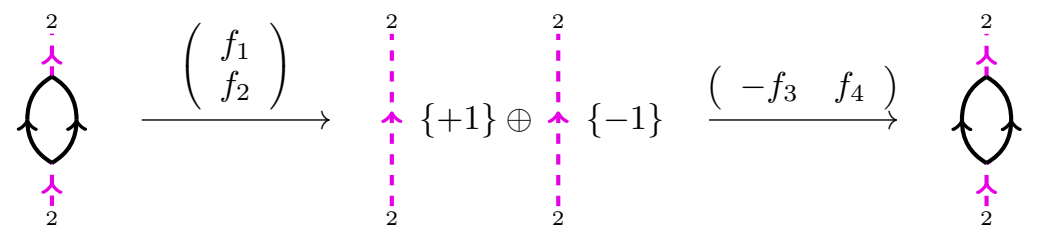

induce isomorphisms in $\mathfrak{W}$-biMod ${ }_{\text {gr }}^{p}$ between web bimodules.

Proof. The statement of one-sided invertibility follows from the top bubble removals 13) (by stacking the foams in the first column on top of the foams in the second column). The invertibility of the foams with a different dot placement follows from the above and the dot migrations (17). The claim that the given morphisms are isomorphisms follows from composing them and using both bubble removals 13 . and (14) as well as the neck cutting relation $(15)$.

The next lemmas say that isotopic webs give isomorphic web modules.

Lemma 4.4. We have locally

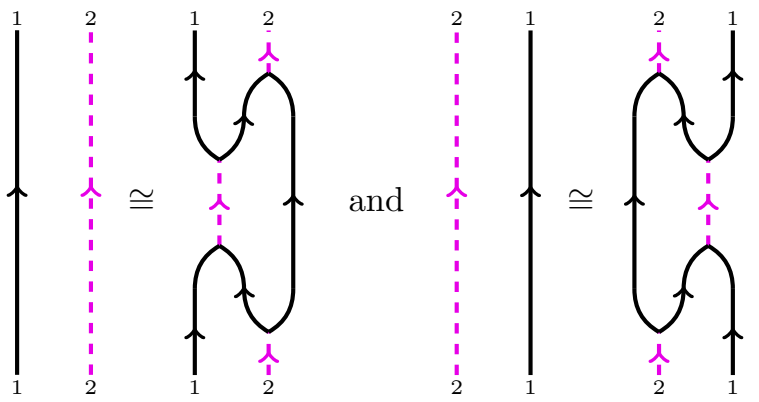

which are isomorphisms in $\mathfrak{W}$-biMod $\mathbf{g r}_{\mathrm{gr}}^{p}$ between web bimodules. 
Proof. The proof is similar to the one of Lemma 4.3 . we can cap the bulge part respectively cup the two straight lines using the evident (undotted) foams. Then the ordinary-to-phantom neck cutting relations (18) provide the isomorphisms.

Lemma 4.5. We have locally (where we use "rectangular" diagrams as in (19))
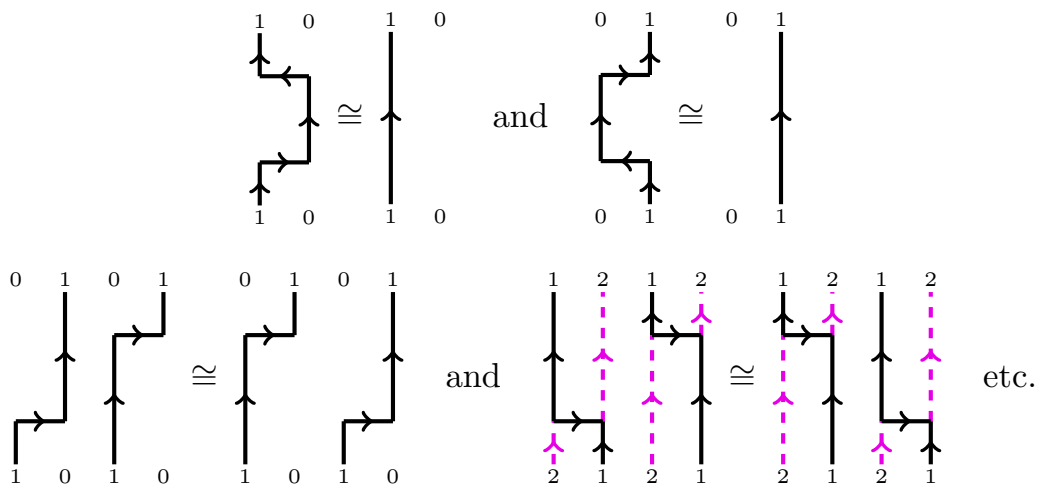

which are isomorphisms in $\mathfrak{W}_{-}$-biMod ${ }_{\mathrm{gr}}^{p}$ between web bimodules. Analogously for other isotopies of webs.

Proof. This is evident.

Given a web $u$, then we denote by $\hat{u}$ the topological web obtained from $u$ by forgetting orientations, labels and phantom edges, i.e. we have locally

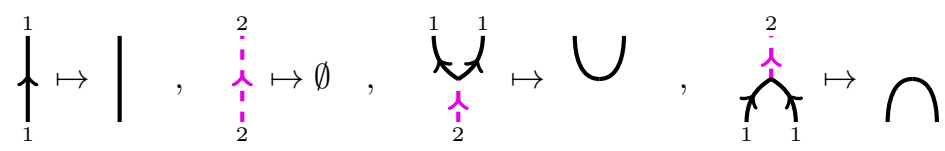

The topological webs are just non-crossing arcs and (closed) circles. We call any web $u$ such that $\hat{u}$ is topologically a circle also a circle. Similarly for webs $u$ such that $\hat{u}$ is topologically a cup, cap or a line. Moreover, we assume that the leftmost non-zero entries of $\Lambda \in \mathrm{bl}^{\diamond}$ and $\vec{k} \in \mathbb{b} \mathbb{1}^{\diamond}$ are at the same position in what follows.

Lemma 4.6. Let $\lambda \in \Lambda$ with $j$ entries equal to $\times$. Consider the cup diagram $\underline{\lambda}$. Assume that $\underline{\lambda}$ does have $\ell^{\prime}$ rays and in total $\ell+\ell^{\prime}-j$ components. Then there exists a cup-ray web $u \in \operatorname{CupRay}(\vec{k})$ such that the entries of $\vec{k} \operatorname{sum}$ up to $2 \ell+\ell^{\prime}$, and such that the topological web $\hat{u}$ is $\underline{\lambda}$.

The sequences $\Lambda \in \mathrm{b} 1^{\diamond}$ and $\vec{k} \in \mathrm{b}^{\diamond}$ are related by the bijection from 38 below.

Proof. Given a cup diagram $\underline{\lambda}$ with $\ell+\ell^{\prime}-j$ components nested in any order, we can generate it using $E_{i}^{(r)}$ 's and $F_{i}^{(r)}$ 's acting on the sequence $\omega_{\ell+\ell^{\prime}}+\omega_{\ell}$. It is clear from 19 that we can open one cup for each entry 2 of $\omega_{\ell+\ell^{\prime}}+\omega_{\ell}$. By using
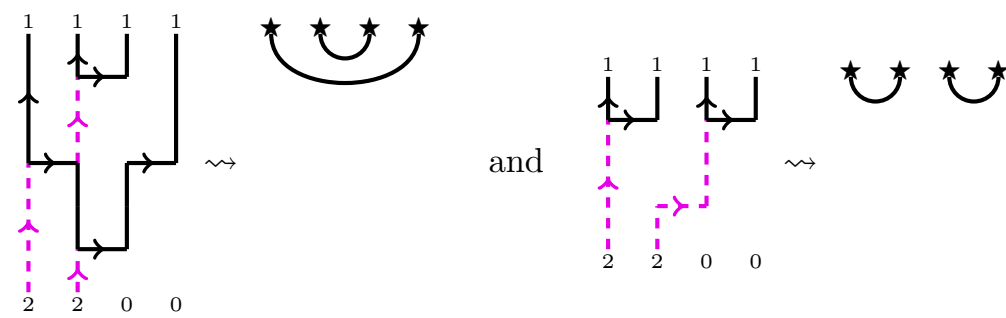

we can nest them and place them next to each other in any order we like (by locally shifting everything in place as above). Last, by using the shifts from (19), we 
can move the remaining entries 1 to form the rays, and move the entries 2 to the positions of the $\times$ symbols while building the cup parts of $\underline{\lambda}$.

Lemma 4.7. For each $\Lambda$-composite matching $\mathbf{t}$, there exists a web $u \in \operatorname{Hom}_{\mathfrak{F}}(\vec{k}, \vec{l})$ such that the topological web $\hat{u}$ is $\mathbf{t}$.

Proof. Mutatis mutandis as in the proof of Lemma 4.6 .

Even if we require a web $u$ to be $F$-generated, there are still several ways to built $\underline{\lambda}$ or $\mathbf{t}$. In order to fix one, we say an $F$-generated web $u$ prefers right to left if, inductively, the component with rightmost right boundary point of $\underline{\lambda}$ or $\mathbf{t}$ is created from the rightmost available 2 , if its a cup, or the rightmost available 1 , if its a ray (using a minimal number of possible moves). We create the right boundary point of a cup before the left. In the whole procedure we avoid creating circles.

Lemma 4.8. In the set-up of Lemma 4.6 or of Lemma 4.7 we can make $u$ unique by requiring it to be $F$-generated and preferring right to left.

Proof. An easy observation shows that we do not need $E_{i}$ 's and $E_{i}^{(2)}$ 's in order to built $\underline{\lambda}$ or $\mathbf{t}$ (this holds more generally, see [37, Lemma 4.9]). Moreover, we can always avoid creating circles. Thus, we obtain a set $F_{\text {gen }}$ of $F$-generated webs such that $\hat{u}$ gives $\underline{\lambda}$ or $\mathbf{t}$. All of these differ by distant commutation relations as in Lemma 4.5 (bottom moves) or Serre relations $\Phi_{\text {Howe }}^{\text {Web }}\left(\left(F_{i+1} F_{i}^{(2)}-F_{i} F_{i+1} F_{i}+F_{i}^{(2)} F_{i+1}\right) \mathbf{1}_{\vec{k}}\right)=0$. One checks that, for fixed $\vec{k} \in \mathfrak{b} \mathbb{1}^{\diamond}$, all Serre relations have only two non-zero terms and that the corresponding pictures are as in Lemmas 4.4 and 4.5 . Hence, there is a unique web in $F_{\text {gen }}$ that prefers right to left which can be shown by induction on the number $m$ of components of $\underline{\lambda}$ or $\mathbf{t}$. This is clear if $m=0$ or $m=1$. For $m>1$ remove the leftmost connected component from $\underline{\lambda}$ or $\mathbf{t}$ and obtain $\underline{\lambda}^{\prime}$ or $\mathbf{t}^{\prime}$ with one connected component less. We can then apply the induction hypothesis and we get a unique web $u^{\prime}$ such that $\hat{u}^{\prime}$ is $\underline{\lambda}^{\prime}$ or $\mathbf{t}^{\prime}$. Since we removed the leftmost component of $\underline{\lambda}$ or of $\mathbf{t}$, we can now just construct $u$ from $u^{\prime}$ (the result is unique due to our conventions for such webs).

Hence, the following definition makes sense.

Definition 4.9. Let $\Lambda \in \mathrm{bl}^{\diamond}$. Given $\lambda \in \Lambda$, we denote by $\mathbf{w}(\lambda)$ the unique web as in Lemma 4.8 , and given a $\boldsymbol{\Lambda}$-composite matching $\mathbf{t}$, we denote the unique web as in Lemma 4.8 by $\mathbf{w}(\boldsymbol{\Lambda}, \mathbf{t})$.

Examples of such webs are given in (37). Moreover, the following assignment

$$
\vec{k} \in \mathrm{bl}^{\diamond} \mapsto \Lambda \in \mathrm{bl}^{\diamond}, \quad \text { via } 0 \mapsto \circ, \quad 1 \mapsto \star, \quad 2 \mapsto \times,
$$

clearly defines a bijection. Here $\circ, \star, \times$ are entries of $\operatorname{seq}(\Lambda)$ and $\Lambda$ is determined demanding that $\Lambda$ is balanced.

The next lemma is important for the calculation of the signs that turn up in the multiplication procedure. For this purpose, fix a circle $C$ in a web $\mathbf{w}(\lambda) \mathbf{w}(\mu)^{*}$ with corresponding circle $C^{\prime}$ in $\underline{\lambda} \bar{\mu}$. For such a circle let nest $(C)$ be the total number of circles $C_{i}^{\text {in }}$ nested in $C$. We denote by ipe $(C)$ the total number of internal phantom edges of $C$ (all such edges that lie in the interior of $C$, but not in the interior of any circle nested in $C$ ) and more generally by

$$
\text { ipe }\left(C-\bigcup_{i=1}^{\text {nest }(C)} C_{i}^{\text {in }}\right)
$$

the total number of internal phantom edges of $C$ after removing $C_{i}^{\text {in }}$ (by using simplifications as isotopies, (36) and (35)). Recalling $\mathrm{d}(\cdot)$ from Definition 3.10 we have the following lemma: 
Lemma 4.10. Given a circle diagram $\underline{\lambda} \bar{\mu}$ (for suitable $\lambda, \mu \in \Lambda$ ) and its associated web $\mathbf{w}(\lambda) \mathbf{w}(\mu)^{*}$. Fix any circle $C^{\prime}$ in $\underline{\lambda} \bar{\mu}$ and denote the associated circle in $\mathbf{w}(\lambda) \mathbf{w}(\mu)^{*}$ by $C$. Then

$$
\begin{aligned}
\operatorname{ipe}(C) & =\frac{1}{4}\left(\mathrm{~d}\left(C^{\prime}\right)+\sum_{i=1}^{\text {nest }(C)} \mathrm{d}\left(C_{\text {in }}^{i}\right)-2+2 \cdot \operatorname{nest}(C)\right), \\
\operatorname{ipe}\left(C-\bigcup_{i=1}^{\text {nest }(C)} C_{i}^{\text {in }}\right) & =\frac{1}{4}\left(\mathrm{~d}\left(C^{\prime}\right)-2\right),
\end{aligned}
$$

where $C_{\text {in }}^{i}$ denotes the counterparts in $\underline{\lambda} \bar{\mu}$ of the circles $C_{\text {in }}^{i}$ of $\mathbf{w}(\lambda) \mathbf{w}(\mu)^{*}$.

Proof. We prove this by induction on the total length of all components in question. Circles $C$ in their easiest form as on the left in 42 below have zero internal phantom edges and $\mathrm{d}(C)=2$, which is the start of our induction. The main observation now is that the move in 36 increases the number of internal phantom edges by one and the length by four (no matter which side of the diagram is the internal part of the circle). This shows the formulas in case that ipe $(C)$ has no nested circles. The general formulas follow similarly, where we note that each $\times$ within $C^{\prime}$ increases its length by 2 and creates a internal phantom edge in $C$.

Example 4.11. The circles $C^{\prime}$ and $C_{\text {in }}^{1}$ as on the right in 42 have $\mathrm{d}\left(C^{\prime}\right)=6$ and $\mathrm{d}\left(C_{\text {in }}^{1}\right)=2$, and its counterpart $C$ has one internal phantom edge after removing the nested circle, while is has two internal phantom edges in total. Circles $C^{\prime}$ of $\mathrm{H}$ or $\mathrm{C}$ shape as in 43 have $\mathrm{d}\left(C^{\prime}\right)=6$ respectively $\mathrm{d}\left(C^{\prime}\right)=10$ and their counterparts have two respectively one internal phantom edge.

4.2. An action of the quantum group $\dot{\mathrm{U}}_{q}\left(\mathfrak{g l}_{\infty}\right)$ and arc diagrams. We obtain now an action of the quantum group on CM (with $\mathbf{C M}$ being as at the end of Subsection 3.4 via the functor

$$
\Phi_{\text {Howe }}^{\text {CM }}: \dot{\mathrm{U}}_{q}\left(\mathfrak{g l}_{\infty}\right) \rightarrow \mathbf{C M},
$$

given on objects by $\Phi_{\text {Howe }}^{\mathrm{CM}}(\vec{k})=\Lambda$ (with associated $\Lambda \in \mathrm{bl} 1^{\diamond}$ as in $(38)$ ) and on identity morphisms by $\Phi_{\text {Howe }}^{\mathrm{CM}}\left(\mathbf{1}_{\vec{k}}\right)=\mathbf{1}_{\Lambda}$. For the generating morphism $E_{i} \mathbf{1}_{\vec{k}}, \Phi_{\text {Howe }}^{\mathrm{CM}}\left(E_{i} \mathbf{1}_{\vec{k}}\right)$ is the bimodule corresponding to the unique local picture in the first row of 31 that fits with $\Lambda$ at position $i$ and $i+1$, or zero if none of them fits, $\Phi_{\text {Howe }}^{\mathrm{CM}}\left(F_{i} \mathbf{1}_{\vec{k}}\right)$ is defined analogously using the local moves in the second row of (31). The divided powers $E_{i}^{(2)} \mathbf{1}_{\vec{k}}$ respectively $F_{i}^{(2)} \mathbf{1}_{\vec{k}}$ are sent to the bimodules for the empty moves in (33) for $2 \alpha_{i}$ respectively $-2 \alpha_{i}$, again only if the block fits at position $i$ and $i+1$, and to zero otherwise. As in Subsection 2.3, all higher divided powers $E_{i}^{(r)} \mathbf{1}_{\vec{k}}, F_{i}^{(r)} \mathbf{1}_{\vec{k}}$ for $r>2$ are sent to zero. By our discussion in the previous subsection, we see that $\Phi_{\text {Howe }}^{\mathrm{CM}}$ is a functor of $\mathbb{K}$-linear categories.

4.3. The cup basis. The goal of this subsection is to define a basis of the morphism spaces on the side of foams which we call the cup basis.

Definition 4.12. Let ${ }_{u}\left(\mathfrak{W}_{\vec{k}}\right)_{v}$ be as in Definition 2.24. Perform the following steps.

(I) Label each circle in $u v^{*}$ by either "no dot" or "dot". Consider all possibilities of labeling the circles in such a way.

(II) For each such possibility we construct a foam $f: \mathbf{1}_{2 \omega_{\ell}} \rightarrow u v^{*}$ via:

- If a circle is in its easiest form (i.e. only one incoming phantom edge and only one outgoing phantom edge), then we locally use the foam at the top right in (34) for circles with label "no dot" and the foam at the bottom right in (34) for circles with label "dot".

- If a circle is more complicated, then we first apply the isomorphisms described in Lemma 4.4 to reduce the circle to its easiest form, then we proceed as before, and finally we rebuild the circle using the inverses as in Lemma 4.4. Then we move the dot to the rightmost facet using (17) 
(the meticulous reader will note that this is ill-defined since there could be more than one rightmost facet, but one can choose any of them: dots passing from one rightmost facet to another always have to move an even number of times across phantom facets, see (36)).

From this we obtain a set of foams $\operatorname{Cup}\left(u v^{*}\right)$ which we call the cup basis of ${ }_{u}\left(\mathfrak{W}_{\vec{k}}\right)_{v}$. Basis elements of this basis are called basis cup foams. This name is justified: by construction, the foams in ${ }_{u}\left(\mathfrak{W}_{\vec{k}}\right)_{v}$ are topological obtained by "cupping off circles in the evident way". Similarly, given $u \in \operatorname{Hom}_{\mathfrak{F}}(\vec{k}, \vec{l})$ we can define a cup basis $\operatorname{Cup}(u)$ of $\mathfrak{W}(u)$ as above with the extra condition that we first close the web $u$ in any possible way.

Lemma 4.13. Let $u, v \in \operatorname{Cup}(\vec{k})$. The set $\operatorname{Cup}\left(u v^{*}\right)$ is a homogeneous, $\mathbb{K}$-linear basis of the space ${ }_{u}\left(\mathfrak{W}_{\vec{k}}\right)_{v}$.

Proof. That $\operatorname{Cup}\left(u v^{*}\right)$ is homogeneous is evident. To show that we get a basis, we use induction on the number $m$ of circles in $u v^{*}$. If $m=0$, then the statement is clear by Lemma 4.2 . So let $m>0$. Choose now any maximally nested circle $C$ (a circle that does not have any nested components) in $u v^{*}$ (we have to choose such a maximally nested circle in order use locally the foams from (34)). Remove it from $u v^{*}$ and obtain a new web $\tilde{u} \tilde{v}^{*}$ with fewer number of circles. By induction, $\operatorname{Cup}\left(\tilde{u} \tilde{v}^{*}\right)$ is a basis of $\tilde{u}\left(\mathfrak{W}_{\vec{k}}\right)_{\tilde{v}}$. Now apply the isomorphism from (35) and create a new circle by taking two copies of the basis elements from $\operatorname{Cup}\left(\tilde{u} \tilde{v}^{*}\right)$ (shifted up by one respectively down by one). The claim of the lemma follows if $C$ was in its easiest form. Otherwise, we rebuild the chosen circle $C$ from the newly created circle using isomorphisms as

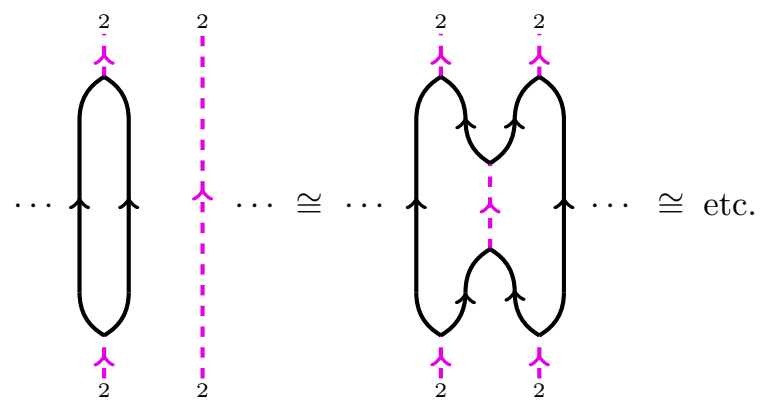

from Lemma 4.4. Finally move dots to the rightmost facets using the dot migrations (17). Thus, the lemma also follows in this case.

Lemma 4.14. Let $u \in \operatorname{Hom}_{\mathfrak{F}}(\vec{k}, \vec{l})$. The set $\operatorname{Cup}(u)$ is a homogeneous, $\mathbb{K}$-linear basis of the web bimodule $\mathfrak{W}(u)$.

Proof. Similar to the proof of Lemma 4.13. the induction start is the same and uses Lemma 4.2. Note that $\mathfrak{W}(u)$ is obtained from $u$ (which has possibly only cups, caps and rays and no circles) by closing the bottom and top in any possible way. Thus, for each such "closure" of $\mathfrak{W}(u)$ we can use the argument from above.

We now match the cup basis $\operatorname{Cup}\left(u v^{*}\right)$ with the basis ${ }_{\lambda} \mathbb{B}^{\circ}(\Lambda)_{\mu}$ defined in (24).

Lemma 4.15. Let $u, v$ be webs such that $u=\mathbf{w}(\lambda)$ and $v=\mathbf{w}(\mu)$. There is an isomorphism of graded $\mathbb{K}$-vector spaces

$$
\Phi_{u v}^{\lambda \mu}:{ }_{u}\left(\mathfrak{W}_{\vec{k}}\right)_{v} \rightarrow{ }_{\lambda}\left(\mathfrak{A}_{\Lambda}^{\mathfrak{F}}\right)_{\mu}
$$

which sends $\operatorname{Cup}\left(u v^{*}\right)$ to ${ }_{\lambda} \mathbb{B}^{\circ}(\Lambda)_{\mu}$ by identifying the cup foams without dots with anticlockwise circles and the foams with dots with clockwise circles. 
Proof. The sets $\operatorname{Cup}\left(u v^{*}\right)$ and ${ }_{\lambda} \mathbb{B}^{\circ}(\Lambda)_{\mu}$ are clearly in bijective correspondence. Moreover, recalling Lemma 3.9 and the shift as in Definition 2.24, we obtain that $\Phi_{u v}^{\lambda \mu}$ is homogeneous, which proves the lemma.

Similarly, we match the cup basis $\operatorname{Cup}(u)$ with the basis $\mathbb{B}^{\circ}(\boldsymbol{\Lambda}, \mathbf{t})$ from $(32)$ :

Lemma 4.16. Let $u$ be a web such that $u=\mathbf{w}(\boldsymbol{\Lambda}, \mathbf{t})$. There is a surjection of graded $\mathbb{K}$-vector spaces

$$
\phi_{u v}^{\lambda \mu}: \mathfrak{W}(u) \rightarrow \mathfrak{A}^{\mathfrak{F}}(\boldsymbol{\Lambda}, \mathbf{t})
$$

which sends $\operatorname{Cup}(u)$ to $\mathbb{B}^{\circ}(\boldsymbol{\Lambda}, \mathbf{t})$ by identifying the basis cup foams without dots with anticlockwise circles and the basis cup foams with dots with clockwise circles.

The statement of Lemma 4.16 can be easily strengthen using Lemma 4.22 . (Morally, the web bimodules are infinite-dimensional in a "stupid way".)

Proof. To show that (41) is indeed a homogeneous, $\mathbb{K}$-linear surjection we can proceed as in the proof of Lemma 4.15 since both bases, $\operatorname{Cup}(u)$ and $\mathbb{B}^{\circ}(\boldsymbol{\Lambda}, \mathbf{t})$, are in the end defined by closing $u$ respectively $\mathbf{t}$ in all possible ways.

Let still $\Lambda \in \mathrm{bl}^{\diamond}$. Given $\lambda, \mu \in \Lambda$, let us denote for $u=\mathbf{w}(\lambda)$ and $v=\mathbf{w}(\mu)$ (and only these)

$$
\mathfrak{W}_{\vec{k}}^{\circ}=\bigoplus_{u, v \in \operatorname{Cup}(\vec{k})}{ }_{u}\left(\mathfrak{W}_{\vec{k}}\right)_{v} \quad \text { and } \quad \mathfrak{W}^{\circ}=\bigoplus_{\vec{k} \in \mathfrak{b} \mathbb{1}} \mathfrak{W}_{\vec{k}} .
$$

The following is a direct consequence of Lemma 4.15 .

Corollary 4.17. For any $\lambda, \mu \in \Lambda$ and $u=\mathbf{w}(\lambda), v=\mathbf{w}(\mu)$ : the isomorphisms $\Phi_{u v}^{\lambda \mu}$ from $(39)$ extend to isomorphisms of graded, $\mathbb{K}$-vector spaces

$$
\Phi_{\vec{k}}^{\Lambda}: \mathfrak{W}_{\vec{k}}^{\circ} \rightarrow \mathfrak{A}_{\Lambda}^{\mathfrak{F}}, \quad \Phi: \mathfrak{W}^{\circ} \rightarrow \mathfrak{A}^{\mathfrak{F}} .
$$

where we identify $\vec{k}$ and $\Lambda$ as in $(38)$.

4.4. Proof of the main result. We deduce now Theorem 4.1 from the following.

Theorem 4.18. The maps from (41) are isomorphisms of graded algebras.

Before we prove Theorem 4.18 in Subsection 4.5, we deduce some consequences, e.g. our main result. Moreover, the identification from Theorem 4.18 allows us to use topological arguments (i.e. foams) to deduce algebraic properties. In particular, we obtain the associativity of the Blanchet-Khovanov algebras.

Corollary 4.19. The multiplication rule from Subsection 3.3 is independent of the order in which the surgeries are performed. This turns $\mathfrak{A}_{\Lambda}^{\mathfrak{F}}$ into a graded, associative, unital algebra. Similar for (the locally unital) algebra $\mathfrak{A}^{\mathfrak{F}}$.

Proof. This follows directly from Theorem 4.18 (note that the stated properties are clear if we work with the web algebra, see Corollary 2.28).

Remark 4.20. Theorem 4.18 leaves the question how the Blanchet-Khovanov algebra $\mathfrak{A}_{\Lambda}^{\mathfrak{F}}$ and Khovanov's original arc algebra $H_{m}$ are related (and thus, how the Blanchet foam construction relates to the Khovanov 17 and Bar-Natan 1 theory using cobordisms). To answer this question, note that the action of $\dot{U}_{q}\left(\mathfrak{g l}_{n}\right)$ from Subsection 4.2 extends to a 2-representation of Khovanov-Lauda's categorification of $\dot{U}_{q}\left(\mathfrak{g l}_{n}\right)$, see [24, Proposition 3.3]. The same holds on the side of Khovanov's arc algebra, see [6, Remark 5.7]. Hence, it follows, for suitable choices of $\Lambda$ and $m$, that $\mathfrak{A}_{\Lambda}^{\mathfrak{F}}$ and $H_{m}$ are (graded) Morita equivalent. This can be deduced from Rouquier's universality theorem, see [33, Proposition 5.6 and Corollary 5.7], compare also to [27. Proposition 5.18]. Since $\mathfrak{A}_{\Lambda}^{\mathfrak{F}}$ and $H_{m}$ are basic algebras, it follows by abstract 
nonsense that $\mathfrak{A}_{\Lambda}^{\mathfrak{F}}$ and $H_{m}$ are isomorphic algebras (in fact, as graded algebras). This approach however does not provide an explicit isomorphism. Such an isomorphism is constructed in [14, Section 4] using a slightly more general framework.

Remark 4.21. Theorem 4.18 gives a way to compute the functorial chain complex (which is a link invariant) defined by Blanchet, see [2, and all its involved maps. Indeed, in our framework $\mathfrak{A}^{\mathfrak{F}}$ comes equipped with an easy to handle basis and all appearing $\mathfrak{A}^{\mathfrak{F}}$-module homomorphisms can explicitly be computed in this basis. This is in contrast to the local action used in 24, Proposition 3.3] to define Blanchet's link homologies, see [24, Subsection 4.1], because it is not a priori clear in their formulation how to do explicit computations (since globally a significant number of non-trivial signs come into play).

Note that we consider webs in $u \in \operatorname{Cup}(\vec{k})$ without imposing any relations. On the "uncategorified level" in the sense of Kuperberg 23, this has to be modified: denote by $\operatorname{Cup}(\vec{k})_{\mathrm{rel}}^{\mathbb{K}(q)}=\left\langle\operatorname{Hom}\left(\mathbf{1}_{2 \omega}, \vec{k}\right)\right\rangle_{\mathbb{K}(q)}$ the $\mathbb{K}(q)$-linear vector space obtained from $\operatorname{Cup}(\vec{k})$ by linearization and modding out by the circle removal relation

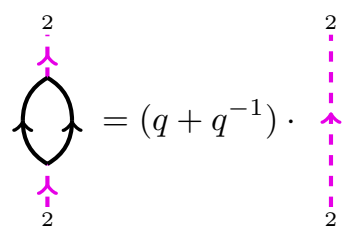

and isotopy relations as in the Lemmas 4.4 and 4.5 . Note that, on the "categorified level" in which we work in the rest of the paper, we do not need to impose these relations since we "lift" them to the isomorphisms from Lemmas 4.3, 4.4 and 4.5 .

Lemma 4.22. Let $\Lambda \in \mathrm{bl}^{\diamond}$ and $\vec{k}$ be its associated element in $\mathfrak{b} \mathbb{1}^{\diamond}$ (see (38)). Then

$$
\{u \in \operatorname{Cup}(\vec{k}) \mid u=\mathbf{w}(\lambda), \lambda \in \Lambda\}
$$

is a $\mathbb{K}(q)$-linear basis of $\operatorname{Cup}(\vec{k})_{\mathrm{rel}}^{\mathbb{K}(q)}$.

Proof. This is clear by the relations imposed on $\operatorname{Cup}(\vec{k})_{\mathrm{rel}}^{\mathbb{K}(q)}$.

We are finally able to prove our main result.

Proof of Theorem 4.1. Instead of taking all webs $u \in \operatorname{Cup}(\vec{k})$, it suffices to take a basis of $\operatorname{Cup}(\vec{k})_{\text {rel }}^{\mathbb{K}(q)}$ and the webs $\mathbf{w}(\lambda)$ form such a basis by Lemma 4.22 . Concretely, the algebras $\mathfrak{W}_{\vec{k}}$ (all webs) and $\mathfrak{W}_{\vec{k}}^{\circ}$ (only basis webs) are graded Morita equivalent (this can be seen similar to [26, Lemma 7.5]) and the statement follows from Theorem 4.18. The identification of the bimodules as graded $\mathbb{K}$-vector spaces is clear by Lemma 4.16 , while the actions agree by Theorem 4.18 and construction of the actions.

4.5. The proof of the graded isomorphism. We now prove Theorem 4.18

Proof of Theorem 4.18. By Lemma 4.15 it suffices to show that $\Phi_{\vec{k}}^{\Lambda}$ is a homomorphism of algebras (since then so is $\Phi$ as well). For this purpose, fix $\lambda, \mu, \nu \in \Lambda$ and set $u=\mathbf{w}(\lambda), v=\mathbf{w}(\mu)$, and $w=\mathbf{w}(\nu)$. We show that any product of two basis cup foams $f \in \operatorname{Cup}\left(u v^{*}\right)$ and $g \in \operatorname{Cup}\left(v w^{*}\right)$ satisfies

$$
\Phi_{u w}^{\lambda \nu}(f g)=\Phi_{u v}^{\lambda \mu}(f) \Phi_{v^{\prime} w}^{\mu^{\prime} \nu}(g), \quad v=v^{\prime}, \mu=\mu^{\prime}
$$

(This is enough since all non-zero multiplications on the side of $\mathfrak{A}_{\Lambda}^{\mathfrak{F}}$ as well as on the side of $\mathfrak{W}_{\vec{k}}$ satisfy $v=v^{\prime}$ and $\mu=\mu^{\prime}$ by definition.) 
In order to do so, we show that each step in the multiplication procedure from Definition 2.24 locally agrees with the one from Subsection 3.3 . There are four topological different situations to check (compare with the cases in Subsection 3.3 introducing the different signs):

(i) Non-nested merge. Two non-nested circles are replaced by one circle.

(ii) Nested merge. Two nested circles are replaced by one circle.

(iii) Non-nested split. One circle is replaced by two non-nested circles.

(iv) Nested split. One circle is replaced by two nested circles.

We will consider these four cases step-by-step and compare the corresponding multiplications rules. In addition to the four cases we will further distinguish the following shapes of the involved underlying webs:

(A) Basic shape. The involved components are as small as possible with the minimal number of phantom facets.

(B) Minimal saddle. While the components themselves are allowed to be of any shape, the involved saddle only includes a single phantom facet.

(C) General case. Both, the shape as well as the saddle, are arbitrary.

We start by comparing the two multiplication rules for the basic shapes first. This will only involve simplified versions of the dot moving signs. The basic shapes for (i), (ii), (iii) and (iv) are the following (with the multiplication step taking place in the marked region)

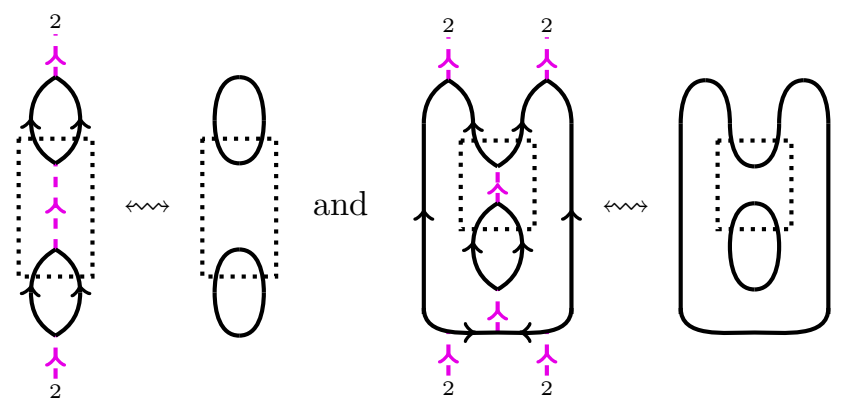

in cases (i) and (ii), and the following H-shape and C-shape
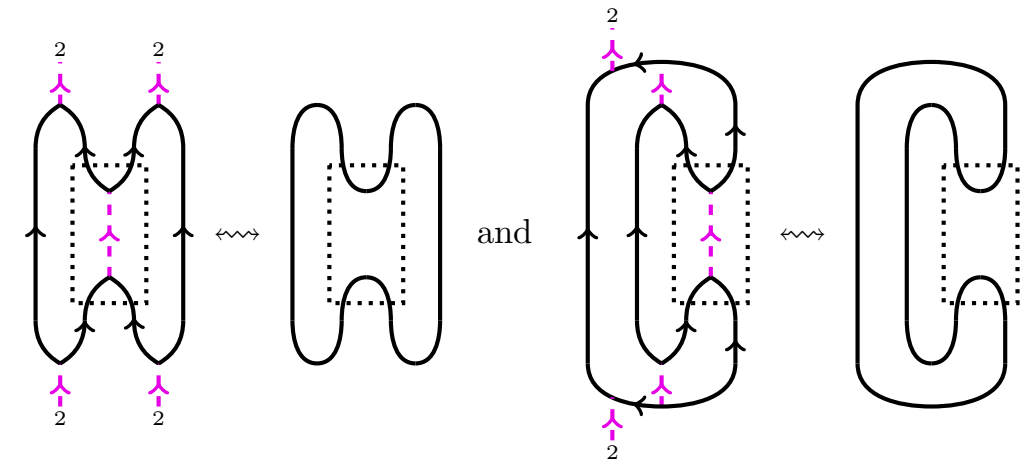

in cases (iii) and (iv). Here we always display both, the web as well as its corresponding arc diagram.

- Non-nested merge - basic shape. In this case, we merge two simple cup webs on the web side and two oriented circles on the arc diagram side. The following table gives the multiplication results in the four possible orientation combinations. 

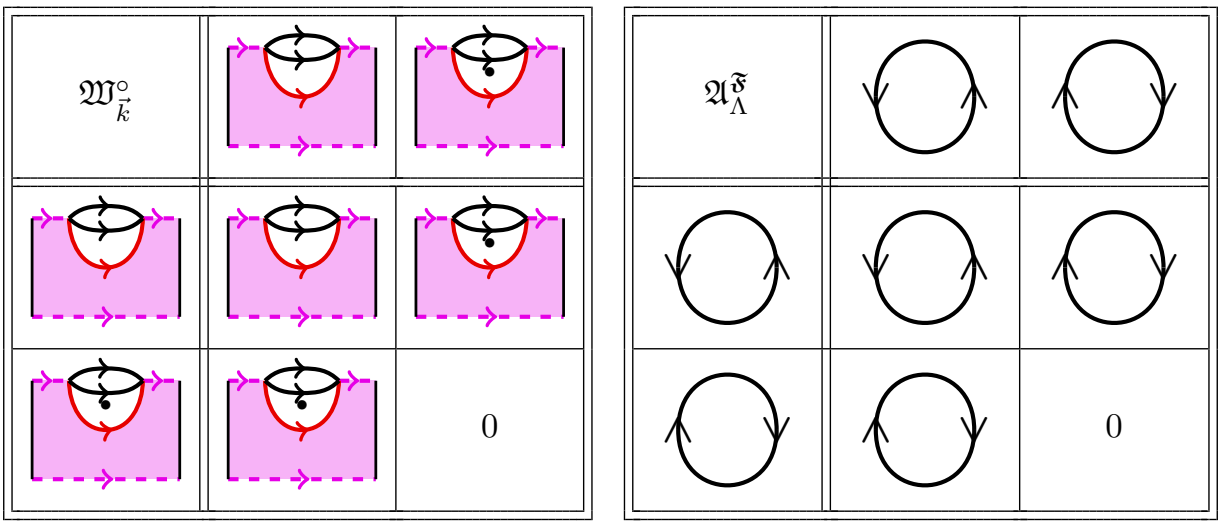

To obtain this table one argues as follows: merging two basis cup foams via a saddle creates a new basis cup foam. Thus, only the position of the dot matters if we rewrite the result in the cup basis. If there is no dot or there are two dots on the new cup foam, then we are done (the latter follows from $(8 p)$. If there is only one dot note that it is automatically on the rightmost facet and we are done as well (no signs). This is precisely as in (27).

- Nested merge - basic shape. This step in case of $\mathfrak{A}_{\Lambda}^{\mathfrak{F}}$ was calculated in 28. In case of $\mathfrak{W}_{\vec{k}}^{\circ}$ this is given by stacking the saddle displayed below on top of a given foam (the second foam displayed below is shown to illustrate the cylinder we want to cut).
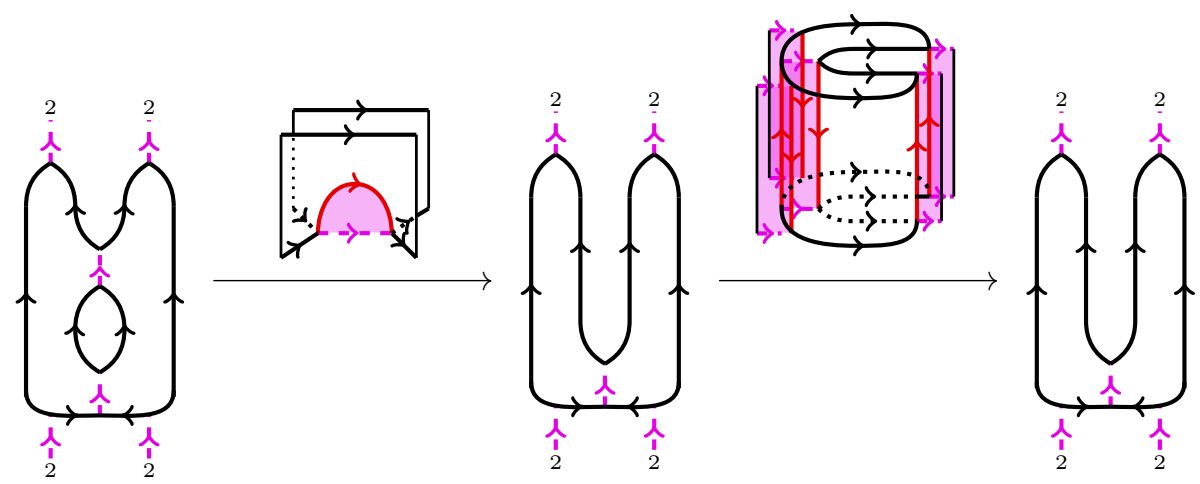

Note now that the difference to the non-nested merge above is that, if a basis cup foam is sitting underneath the leftmost picture, then the end result is topological not a basis cup foam. In order to turn the result into a basis cup foam, we apply 15 to the cylinder illustrated above. Here we have to use 16 first, which gives an overall sign. After neck cutting the cylinder we create a "bubble" (recalling that a basis cup foam is sitting underneath) with two internal phantom facets in the bottom part of the picture. By (18), we can remove the phantom facets with the cost of a sign and create an "honest" bubble instead. Thus, by (14), only the term in 15 with the dot on the bottom survives (with a sign). By (13) the remaining bubble evaluates to -1 . Hence, we get in total four overall signs which is the same as no extra sign. The dots behave as in the table above, since before the neck cut we can move any of them to the top and thus they do not interfere with the above procedure. Thus, using (17), we get the same result as in 28). 
- Non-nested split - basic shape. This step in case of $\mathfrak{A}_{\Lambda}^{\mathfrak{F}}$ was calculated in $(29)$. For $\mathfrak{W}_{\vec{k}}^{\circ}$ the multiplication is given by the composition of the following foams.
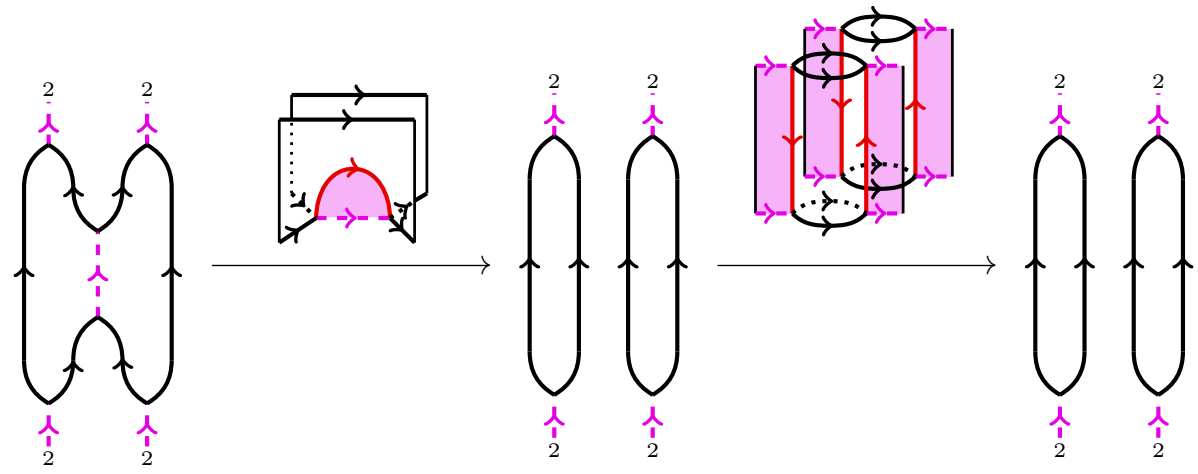

As before, we can use the neck cutting (15) on the cylinder that corresponds to the right circle (we could also choose the other one and slightly change the steps below). The result is precisely as in 29$)$ :

- If the original basis cup foams sitting underneath has no dots, then the one with the dot on the rightmost circle gets no sign (the dot is automatically on the rightmost facet), and the other does not as well (the dot from the neck cutting needs to pass one phantom facets to move to the right).

- If the original basis cup foams sitting underneath has already a dot, then only the positive term in 15 survives and the dots are already in the rightmost positions.

- In both cases, the resulting foam is topological not a basis cup foam, but using (18) once reduces it to a basis cup foam, giving an overall sign.

- Nested split - basic shape. This step in case of $\mathfrak{A}_{\Lambda}^{\mathfrak{F}}$ was calculated in $(30)$. In case of $\mathfrak{W}_{\vec{k}}^{\circ}$ we again give the composite of foams that we stack on top of each other for the multiplication.
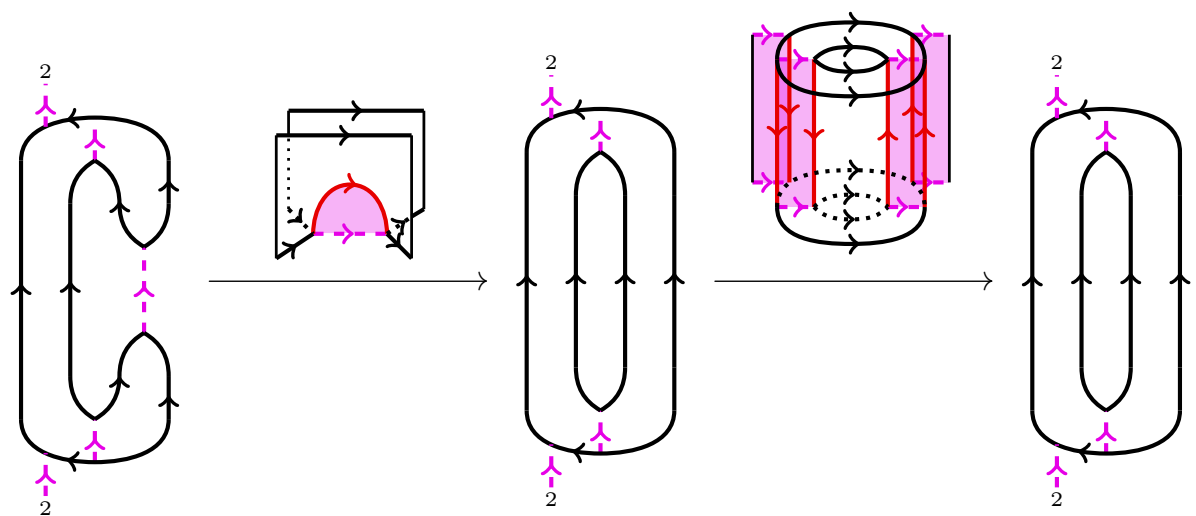

Again we can apply neck cutting. This time to the internal cylinder in the second foam between the middle web and the rightmost web connecting the two nested circles that we can cut using (15). The result is precisely as in (30):

- If the original basis cup foams sitting underneath has no dots, then the one with the dot on the non-nested circle gets a sign (the dot is automatically on the rightmost facet), while the other does not (the dot created in the neck cutting needs to pass two phantom facets to get to the right). 
- If the original basis cup foams sitting underneath has already a dot, then only the positive term in 15 survives and the dots are already in the rightmost positions.

- In both cases, the resulting foam is already topological a cup basis foam and nothing needs to be done anymore.

All other situations (i.e. general shapes) are similar to the ones discussed, but with the main difference that dots need to be shifted to the rightmost facets, some (intermediate) results might not be in the topological form of a basis cup foam and one needs to take care of saddles with a possible number of additional internal phantom facets as in (21). These three facts together explain the signs turning up in the multiplication described in Subsection 3.3 with the first one corresponding to the dot moving sign as in 25 , the second corresponding to the topological signs as in (26) and the latter to the saddle sign as in (26).

Thus, we next deal with the minimal saddle situation as for example in (22). In those cases $s(\gamma)=1$ on the side of $\mathfrak{A}_{\Lambda}^{\mathfrak{F}}$. This will introduce the topological signs and simplified versions (for $s(\gamma)=1$ ) of the saddle signs.

- Non-nested merge - minimal saddle. This is topologically the same as in non-nested merge - basic shape, since the resulting foam will already be a basis cup foam. Thus, the only sign comes from moving dots to the right which matches the dot moving sign turning up in the multiplication on the side of $\mathfrak{A}_{\Lambda}^{\mathfrak{F}}$ in $(25)$.

- Nested merge - minimal saddle. Assume now that we merge a circle $C^{\text {out }}$ with some nested circle $C^{\text {in }}$ inside of it.

The dot moving is as above in non-nested merge - minimal saddle and gives the same sign as for $\mathfrak{A}_{\Lambda}^{\mathfrak{F}}$. The difference to the nested merge in the basic shape is that we have to bring the resulting foam in the topological form of a basis cup foam. To this end, we can proceed as in nested merge - basic shape and cut the same cylinder as there. We first note that neither the dots which are already on the foam sitting underneath nor the internal circles in $C^{\text {out }}$ which are different from $C^{\text {in }}$ matter: we can topologically move them "away from the local picture".

Thus, to simplify a little bit, assume that $C^{\text {in }}$ is the only circle nested in $C^{\text {out }}$ and there are no dots. Following the procedure given as in nested merge - basic shape above, we see that the only things of importance are signs that come from cutting the cylinder with possible internal phantom facets and evaluating the "bubble" with possible more than one internal phantom facets. Indeed, what matters is the number of times we need to use $(16)$ in the cutting procedure of the cylinder and the number of times we need to use (18) in the bursting of the "bubble" (the rest stays the same as before in nested merge - basic shape). Now, the number of times we need to apply 16 is ipe $\left(C^{\text {out }}\right)-1$ (the -1 comes in because we apply a saddle which removes one of the internal phantom edges of the starting picture) and the number of times we need to apply $\sqrt{18}$ is ipe $\left(C^{\text {out }}-C^{\text {in }}\right)$. By Lemma 4.10 , we obtain that

$$
\begin{aligned}
(-1)^{\mathrm{ipe}\left(C^{\text {out }}\right)-1+\mathrm{ipe}\left(C^{\text {out }}-C^{\text {in }}\right)} & =(-1)^{\frac{1}{4}\left(2\left(\mathrm{~d}\left(C_{\text {out }}\right)-2\right)+\mathrm{d}\left(C_{\text {in }}\right)-2\right)} \\
& =-(-1)^{\frac{1}{4}\left(\mathrm{~d}\left(C_{\text {in }}\right)-2\right)} \cdot(-1)^{1},
\end{aligned}
$$

where $C_{\text {out }}$ and $C_{\text {in }}$ are the cup diagram counterparts of $C^{\text {out }}$ and $C^{\text {in }}$. This is precisely the same sign turning up on the side of $\mathfrak{A}_{\Lambda}^{\mathfrak{F}}$ (compare to 26) with $\left.s_{\Lambda}(\gamma)=1\right)$.

The case where $C^{\text {out }}$ has several nested components, is similar since all nested components of $C^{\text {out}}$, which are not $C^{\text {in }}$, increase the number of times we need to use (16) in the same way as the number of times we need to use (18) (hence, no change modulo 2). Again, this matches the side of $\mathfrak{A}_{\Lambda}^{\mathfrak{F}}$ in 26 . 
- Non-nested split - minimal saddle. The dot moving is as before. Observe now that we do not have an extra sign turning up although the resulting foam is not in the topological shape of a basis cup foam. To see this, we first simplify by assuming that the circle $C$ which is split does not contain any nested components. Using the same cutting as in non-nested split - basic shape, we have signs coming from squeezing cylinders and simplifying "bubbles" (similar as above in nested merge - minimal saddle). But the number of times we need to apply 16 in this case is now the same as the number of times one has to apply 18 , namely ipe $(C)-1$. Thus, again no change modulo 2 . The case with nested components in $C$ is now analogously as above in nested merge - minimal saddle since we can move dots and nested circles "away". As before, this increases the number of times we need to use (16) in the same way as the number of times we need to use (18) (hence, no change mod 2). Thus, we do not get an extra overall sign as in case of $\mathfrak{A}_{\Lambda}^{\mathfrak{F}}$ (see Subsection 3.3 .3 non-nested split case).

- Nested split - minimal saddle. Moving dots is again as before. Furthermore, again, as in nested merge - minimal saddle, we need to topologically manipulate the resulting foam until it is in basis cup foam shape. We can proceed as before in nested split - basic shape and, similar as above in nested merge - minimal saddle, we pick up signs coming from cylinder cuts and bubble removals. In fact, the total sign can be calculated analogously as in nested merge - minimal saddle (and is the same as there). Again, this matches the side of $\mathfrak{A}_{\Lambda}^{\mathfrak{F}}$ (see Subsection 3.3.3 nested split case).

- Non-nested merge - general case. In fact, nothing changes compared to the discussion in non-nested merge - minimal saddle, since dots passing a saddle always pass an odd number of phantom facets (compare to (21)) and the resulting foams are topological already basis cup foams.

- Nested merge - general case. The dot moving stays as before. The only thing that changes in contrast to nested merge - minimal saddle is that we obtain

$$
\begin{aligned}
(-1)^{\text {ipe }\left(C^{\text {out }}\right)-s+i p e}\left(C^{\text {out }}-C^{\text {in }}\right) & =(-1)^{\frac{1}{4}\left(2\left(\mathrm{~d}\left(C_{\text {out }}\right)-2\right)+\mathrm{d}\left(C_{\text {in }}\right)-2\right)} \cdot(-1)^{s(\gamma)-1} \\
& =-(-1)^{\frac{1}{4}\left(\mathrm{~d}\left(C_{\text {in }}\right)-2\right)} \cdot(-1)^{s(\gamma)} .
\end{aligned}
$$

within the topological re-writing procedure instead of the formula from (44). Thus, this matches the side of $\mathfrak{A}_{\Lambda}^{\mathfrak{F}}($ see $(26)$ ).

- Non-nested split - general case. Again, the dot moving stays as before. The difference to non-nested split - minimal saddle is that we have to apply $(18)$ $s$-times instead of once, which gives the sign turning up for $\mathfrak{A}_{\Lambda}^{\mathfrak{F}}$ (see Subsection 3.3 .3 non-nested split case).

- Nested split - general case. There is no difference to the arguments given in nested split - minimal saddle. Again, this matches the side of $\mathfrak{A}_{\Lambda}^{\mathfrak{F}}$ (see Subsection 3.3.3 nested split case).

Hence, in each case the topological multiplication agrees with the algebraically defined one. This concludes the proof.

\section{REFERENCES}

[1] D. Bar-Natan. Khovanov's homology for tangles and cobordisms. Geom. Topol., 9:1443-1499, 2005. URL: http://arxiv.org/abs/math/0410495 doi:10.2140/gt.2005.9.1443

[2] C. Blanchet. An oriented model for Khovanov homology. J. Knot Theory Ramifications, 19(2):291-312, 2010. URL: http://arxiv.org/abs/1405.7246, doi:10.1142/ S0218216510007863 
[3] C. Blanchet, N. Habegger, G. Masbaum, and P. Vogel. Topological quantum field theories derived from the Kauffman bracket. Topology, 34(4):883-927, 1995. doi:10.1016/0040-9383(94) 00051-4

[4] J. Brundan and C. Stroppel. Highest weight categories arising from Khovanov's diagram algebra I: cellularity. Mosc. Math. J., 11(4):685-722, 821-822, 2011. URL: http://arxiv.org/ abs/0806.1532

[5] J. Brundan and C. Stroppel. Highest weight categories arising from Khovanov's diagram algebra II: Koszulity. Transform. Groups, 15(1):1-45, 2010. URL: http://arxiv.org/abs/0806.3472, doi:10.1007/s00031-010-9079-4

[6] J. Brundan and C. Stroppel. Highest weight categories arising from Khovanov's diagram algebra III: category $\mathcal{O}$. Represent. Theory, 15:170-243, 2011. URL: http://arxiv.org/abs/ 0812.1090 doi:10.1090/S1088-4165-2011-00389-7

[7] J. Brundan and C. Stroppel. Highest weight categories arising from Khovanov's diagram algebra IV: the general linear supergroup. J. Eur. Math. Soc. (JEMS), 14(2):373-419, 2012. URL: http://arxiv.org/abs/0907.2543, doi:10.4171/JEMS/306

[8] J. Brundan and C. Stroppel. Gradings on walled Brauer algebras and Khovanov's arc algebra. Adv. Math., 231(2):709-773, 2012. URL: http://arxiv.org/abs/1107.0999 doi:10.1016/j. aim.2012.05.016

[9] S. Cautis, J. Kamnitzer, and S. Morrison. Webs and quantum skew Howe duality. Math. Ann., 360(1-2):351-390, 2014. URL: http://arxiv.org/abs/1210.6437, doi:10.1007/ s00208-013-0984-4

[10] D. Clark, S. Morrison, and K. Walker. Fixing the functoriality of Khovanov homology. Geom. Topol., 13(3):1499-1582, 2009. URL: http://arxiv.org/abs/math/0701339, doi:10.2140/gt. 2009.13.1499

[11] M. Ehrig and C. Stroppel. 2-row Springer fibres and Khovanov diagram algebras for type D. Canad. J. Math., 68(6):1285-1333, 2016. URL: http://arxiv.org/abs/1209.4998 doi: 10.4153/CJM-2015-051-4.

[12] M. Ehrig and C. Stroppel. Diagrammatic description for the categories of perverse sheaves on isotropic Grassmannians. Selecta Math. (N.S.), 22(3):1455-1536, 2016. URL: http://arxiv org/abs/1511.04111, doi:10.1007/s00029-015-0215-9

[13] M. Ehrig and C. Stroppel. On the category of finite-dimensional representations of $\operatorname{OSp}(r \mid 2 n)$ : Part I. In Representation Theory - Current Trends and Perspectives, EMS Ser. Congr. Rep., pages 109-170. Eur. Math. Soc., Zürich, 2016. URL: https://arxiv.org/abs/1607.04034

[14] M. Ehrig, C. Stroppel, and D. Tubbenhauer. Generic $\mathfrak{g l}_{2}$-foams, web and arc algebras. URL: http://arxiv.org/abs/1601.08010

[15] M. Ehrig, D. Tubbenhauer, and A. Wilbert. Singular TQFTs, foams and type D arc algebras. URL: http://arxiv.org/abs/1611.07444

[16] M. Jacobsson. An invariant of link cobordisms from Khovanov homology. Algebr. Geom. Topol., 4:1211-1251 (electronic), 2004. URL: http://arxiv.org/abs/math/0206303 doi:10. 2140/agt.2004.4.1211.

[17] M. Khovanov. A categorification of the Jones polynomial. Duke Math. J., 101(3):359-426, 2000. URL: http://arxiv.org/abs/math/9908171 doi:10.1215/S0012-7094-00-10131-7

[18] M. Khovanov. A functor-valued invariant of tangles. Algebr. Geom. Topol., 2:665-741, 2002. URL: http://arxiv.org/abs/math/0103190 doi:10.2140/agt.2002.2.665

[19] M. Khovanov. sl(3) link homology. Algebr. Geom. Topol., 4:1045-1081, 2004. URL: http: //arxiv.org/abs/math/0304375 doi:10.2140/agt.2004.4.1045

[20] M. Khovanov and A.D. Lauda. A categorification of quantum $\mathfrak{s l}_{n}$. Quantum Topol., 1(1):1-92, 2010. URL: http://arxiv.org/abs/0807.3250 doi:10.4171/QT/1

[21] M. Khovanov and L. Rozansky. Matrix factorizations and link homology. Fund. Math., 199(1):1-91, 2008. URL: http://arxiv.org/abs/math/0401268 doi:10.4064/fm199-1-1

[22] J. Kock. Frobenius algebras and 2D topological quantum field theories, volume 59 of London Mathematical Society Student Texts. Cambridge University Press, 2004.

[23] G. Kuperberg. Spiders for rank 2 Lie algebras. Comm. Math. Phys., 180(1):109-151, 1996. URL: http://arxiv.org/abs/q-alg/9712003

[24] A.D. Lauda, H. Queffelec, and D.E.V. Rose. Khovanov homology is a skew Howe 2representation of categorified quantum $\mathfrak{s l}(m)$. Algebr. Geom. Topol., 15(5):2517-2608, 2015. URL: http://arxiv.org/abs/1212.6076, doi:10.2140/agt.2015.15.2517.

[25] G. Lusztig. Introduction to quantum groups. Modern Birkhäuser Classics. Birkhäuser/Springer, New York, 2010. Reprint of the 1994 edition. doi:10.1007/978-0-8176-4717-9

[26] M. Mackaay. The $\mathfrak{s l}_{n}$-web algebras and dual canonical bases. J. Algebra, 409:54-100, 2014. URL: http://arxiv.org/abs/1308.0566, doi:10.1016/j·jalgebra.2014.02.036

[27] M. Mackaay, W. Pan, and D. Tubbenhauer. The $\mathfrak{s l}_{3}$-web algebra. Math. Z., 277(1-2):401-479, 2014. URL: http://arxiv.org/abs/1206.2118 doi:10.1007/s00209-013-1262-6 
[28] M. Mackaay, M. Stošić, and P. Vaz. $\mathfrak{s l}_{N}$-link homology $(N \geq 4)$ using foams and the KapustinLi formula. Geom. Topol., 13(2):1075-1128, 2009. URL: http://arxiv.org/abs/0708.2228. doi:10.2140/gt.2009.13.1075

[29] H. Queffelec and David E. V. Rose. The $\mathfrak{s l}_{n}$ foam 2-category: a combinatorial formulation of Khovanov-Rozansky homology via categorical skew Howe duality. Adv. Math., 302:1251-1339, 2016. URL: http://arxiv.org/abs/1405.5920 doi:10.1016/j.aim.2016.07.027

[30] J. Rasmussen. Khovanov's invariant for closed surfaces. URL: http://arxiv.org/abs/math/ 0502527 .

[31] L.-H. Robert. A characterization of indecomposable web modules over Khovanov-Kuperberg algebras. Algebr. Geom. Topol., 15(3):1303-1362, 2015. URL: http://arxiv.org/abs/1309. 2793, doi:10.2140/agt.2015.15.1303

[32] L.-H. Robert. A large family of indecomposable projective modules for the KhovanovKuperberg algebras of $\mathfrak{s l}_{3}$-webs. J. Knot Theory Ramifications, 22(11):1350062, 25, 2013. URL: http://arxiv.org/abs/1207.6287, doi:10.1142/S0218216513500624

[33] R. Rouquier. 2-Kac-Moody algebras. URL: http://arxiv.org/abs/0812.5023

[34] A. Sartori. Categorification of tensor powers of the vector representation of $\mathrm{U}_{q}(\mathfrak{g l}(1 \mid 1))$. Selecta Math. (N.S.), 22(2):669-734, 2016. URL: http://arxiv.org/abs/1305.6162 doi: 10.1007/s00029-015-0202-1

[35] C. Stroppel. TQFT with corners and tilting functors in the Kac-Moody case. URL: http: //arxiv.org/abs/math/0605103

[36] D. Tubbenhauer. $\mathfrak{s l}_{3}$-web bases, intermediate crystal bases and categorification. J. Algebraic Combin., 40(4):1001-1076, 2014. URL: http://arxiv.org/abs/1310.2779, doi: 10.1007/s10801-014-0518-5

[37] D. Tubbenhauer. $\mathfrak{s l}_{n}$-webs, categorification and Khovanov-Rozansky homologies. URL: http: //arxiv.org/abs/1404.5752

M.E.: School of Mathematics \& Statistics, Carslaw Building, University of Sydney, NSW 2006, Australia

E-mail address: michael.ehrig@sydney.edu.au

C.S.: Mathematisches Institut, Universität Bonn, Endenicher Allee 60, Room 4.007, 53115 Bonn, Germany

E-mail address: stroppel@math.uni-bonn.de

D.T.: Mathematisches Institut, Universität Bonn, Endenicher Allee 60, Room 1.003, 53115 Bonn, Germany

E-mail address: dtubben@math.uni-bonn.de 